\title{
Relativistic MHD simulations of extragalactic jets ${ }^{\star}$
}

\author{
T. Leismann ${ }^{1}$, L. Antón ${ }^{2}$, M. A. Aloy ${ }^{1}$, E. Müller ${ }^{1}$, J. M. Martí ${ }^{2}$, J. A. Miralles ${ }^{3}$, and J. M. Ibáñez ${ }^{2}$ \\ 1 Max-Planck-Institut für Astrophysik, Postfach 1312, 85741 Garching, Germany \\ e-mail: maa@mpa-garching.mpg.de \\ 2 Departamento de Astronomía y Astrofísica, Universidad de Valencia, 46100 Burjassot, Spain \\ 3 Departamento de Física Aplicada, Universidad de Alicante, Ap. Correus 99, 03080 Alacant, Spain
}

Received 10 December 2004 / Accepted 5 March 2005

\begin{abstract}
We have performed a comprehensive parameter study of the morphology and dynamics of axisymmetric, magnetized, relativistic jets by means of numerical simulations. The simulations have been performed with an upgraded version of the GENESIS code which is based on a second-order accurate finite volume method involving an approximate Riemann solver suitable for relativistic ideal magnetohydrodynamic flows, and a method of lines. Starting from pure hydrodynamic models we consider the effect of a magnetic field of increasing strength (up to $\beta \equiv|b|^{2} / 2 p \approx 3.3$ times the equipartition value) and different topology (purely toroidal or poloidal). We computed several series of models investigating the dependence of the dynamics on the magnetic field in jets of different beam Lorentz factor and adiabatic index.

We find that the inclusion of the magnetic field leads to diverse effects which contrary to Newtonian magnetohydrodynamics models do not always scale linearly with the (relative) strength of the magnetic field. The relativistic models show, however, some clear trends. Axisymmetric jets with toroidal magnetic fields produce a cavity which consists of two parts: an inner one surrounding the beam which is compressed by magnetic forces, and an adjacent outer part which is inflated due to the action of the magnetic field. The outer border of the outer part of the cavity is given by the bow-shock where its interaction with the external medium takes place. Toroidal magnetic fields well below equipartition $(\beta=0.05)$ combined with a value of the adiabatic index of $4 / 3$ yield extremely smooth jet cavities and stable beams.

Prominent nose cones form when jets are confined by toroidal fields and carry a high Poynting flux $\left(\sigma \equiv \mid b^{2} / \rho>0.01\right.$ and $\beta \geq 1$ ). In contrast, none of our models possessing a poloidal field develops such a nose cone. The size of the nose cone is correlated with the propagation speed of the Mach disc (the smaller the speed the larger is the size). If two models differ only by the adiabatic index, jets having smaller adiabatic indices tend to develop smaller nose cones.
\end{abstract}

Key words. magnetohydrodynamics (MHD) - methods: numerical - relativity - galaxies: active - galaxies: jets

\section{Introduction}

The presence of magnetic fields in relativistic fluids is ubiquitous among the most luminous objects in the universe like, e.g., active galactic nuclei (AGNs), quasars, gamma-ray bursts, $\mathrm{X}$-ray binaries, core-collapse supernovae, etc. In the specific case of jets originating from AGNs, the observation of nonthermal synchrotron radiation (see, e.g., Ferrari 1998, for a review) requires the presence of a magnetic field in which relativistic electrons gyroradiate. The high degree of polarization observed in many AGN sources (e.g., Gabuzda et al. 2000) indicates that magnetic fields are not randomly oriented but posses some large scale structure (at least at parsec scales).

Disentangling the intrinsic structure of the magnetic field is still an observational challenge because of the finite spatial resolution of the interferometric beams used in observations

^ Appendices are only available in electronic form at http://www.edpsciences.org of AGN jets. At parsec scales a variety of magnetic field configurations are observed including predominantly toroidal ones (e.g., 0954 + 658: Gabuzda \& Cawthorne 1996; $1803+784$ : $0823+033$ and $1749+701$ : Gabuzda \& Pushkarev 2001), configurations with alternating orthogonal, alternating aligned and predominantly poloidal fields along the jet (e.g., OJ 287: Gabuzda \& Gómez 2001; 1418 + 546: Gabuzda 2003), and even helical field configurations $(1055+018$ : Attridge et al. 1999; 0820 + 225: Gabuzda et al. 2001; $0745+241$ : Pushkarev \& Gabuzda 2001; 1652 + 398: Gabuzda 2003). Different magnetic field topologies are also observed at kiloparsec scales ranging from fields which are mostly aligned with the jet (e.g., 3C 120: Walker et al. 1987; NGC 4258: Krause \& Löhr 2004) to fields which are oriented preferentially perpendicular (toroidal) to the jet axis (e.g., 3C 449: Kigure et al. 2004). Other more complex structures are observed, too (e.g., Laing \& Bridle 2002). Furthermore, there are indications that the intergalactic and interstellar medium the AGN jets are propagating 
into can be threaded with large scale magnetic fields of some microgauss (e.g., Kim et al. 1990; Crusius-Waetzel et al. 1990; Taylor \& Perley 1993). This variety of ordered magnetic field structures most likely reflects different formation and evolutionary processes. For example, magnetized jets with a dominant toroidal field component can be produced by winding up an initial field which has a significant longitudinal component due to the rotation of the central AGN engine (e.g., Nakamura et al. 2001; Lovelace et al. 2002; Tsinganos \& Bogovalov 2002; Lynden-Bell 2003).

Relativistic magnetized jets from AGNs can be described in the framework of ideal relativistic magnetohydrodynamics (RMHD). Among the first attempts to simulate the corresponding nonlinear, time dependent and multidimensional RMHD equations we mention here that of Evans \& Hawley (1988), who using a two-dimensional finite difference code investigated several problems involving general relativistic magnetohydrodynamic accretion flows onto a black hole. More importantly, these authors also proposed a new numerical technique called constrained transport (CT) for evolving the induction equation while maintaining vanishing divergence of the magnetic field down to machine roundoff error. Later on van Putten (1993, 1996) performed the first axisymmetric simulations of RMHD jets having moderately small Lorentz factors $(\lessgtr 3)$. Koide \& coworkers (Koide et al. 1996; Koide 1997) simulated 2D RMHD slab jets being restricted, however, to rather low spatial resolution, short evolution times, and small Lorentz factors $(\lesssim 4.6)$. This study was extended to 3D jets by Nishikawa $\&$ coworkers (Nishikawa et al. 1997, 1998). Komissarov developed a high-resolution, shock-capturing, second-order accurate RMHD code (Komissarov 1999a) which he used to simulate 2D jets possessing toroidal fields (Komissarov 1999b) and the jet-torus structure observed in the Crab nebula (Komissarov \& Lyubarsky 2003, 2004, see also Del Zanna et al. 2004). Jet formation has been studied using general relativistic magnetohydrodynamic (GRMHD) simulations based on a simplified Total Variation Diminishing scheme ignoring the evolution of the constraint $\nabla \cdot \boldsymbol{B}=0$. Koide et al. $(1998,1999,2000,2002)$ and Koide (2003) modeled the formation of axisymmetric jets in a system consisting of an accretion disk and a rotating black hole. The simulations, which cover a few rotational periods of the black hole, show the formation of a relativistic outflow with a Lorentz factor $W \approx 2$. A similar study but without any symmetry restrictions was carried by Nishikawa et al. (2002, 2003). Jet formation in the context of gamma-ray burst progenitors was considered by Mizuno et al. (2004a,b) using a GRMHD code (and no specific measures to maintain $\nabla \cdot \boldsymbol{B}=0$ ). The evolution of magnetized accretion tori around rotating black holes was investigated in axisymmetry by Gammie et al. (2003) and De Villiers \& Hawley (2003); De Villiers \& Hawley (2003) including a CT algorithm to keep the magnetic field divergence-free. While Gammie et al. (2003) used a Godunovtype conservative scheme, De Villiers \& Hawley (2003) employed a ZEUS-like (i.e., non conservative) scheme to integrate the GRMHD equations.

In previous $2 \mathrm{D}$ RMHD simulations relativistic jets only served the purpose of demonstrating the capabilities of the RMHD code (e.g., Komissarov 1999a, in slab geometry;
Del Zanna et al. 2003, assuming axial symmetry and using cylindrical coordinates), or the simulations only covered a very limited range of jet parameters (e.g., in Komissarov 1999b, only two models were considered both involving only toroidal fields). In the following we present the first comprehensive parameter study of the morphology and dynamics of magnetized relativistic axisymmetric jets including both toroidal and poloidal field configurations of different strength, and beam plasmas of different adiabatic index. Preliminary results of our research can be found in Leismann et al. (2004).

The paper is organized as follows. The basic equations and the numerical algorithm used in the code are discussed in Sect. 2. As we assume cylindrical symmetry in our simulations of relativistic magnetized jets, we give the explicit form of the corresponding equations in the Appendix A. Various tests our code has passed successfully are described in Appendix B. In Sect. 3 we discuss the simulation setup and the model parameters, and in Sect. 4 we present the results of our study. Finally, in Sect. 5 we discuss and interpret our findings, and point out some limitations of our approach.

\section{Numerical method}

In this section we describe the details of the numerical algorithm which we used to integrate the equations of ideal relativistic magnetohydrodynamics (Sect. 2.1). The numerical method is implemented on an upgraded version of GENESIS (Aloy et al. 1999b) and relies on the modular structure of its predecessor. As GENESIS, it is based on a directional-splitting, Godunov-type, finite-volume method, a series of intercell reconstruction routines and a method of lines for the time advance. The new code is second order accurate both in space and time, and relies on a constrained transport method (mainly based on the developments of Ryu et al. 1998) in order to keep (to machine precision) the magnetic field divergencefree throughout a simulation. Unlike GENESIS, the numerical fluxes at the cell interfaces are computed employing an HLL-type solver (Sect. 2.3.1) which uses as the maximum and minimum signal speeds some accurate estimates which are given in Sect. 2.2.1 together with the generic spectral decomposition and properties of the RMHD system of conservation laws. The combination of an HLL-like solver along with a high order spatial reconstruction and a consistently high order method to keep the divergence-free property of the magnetic field was proven to be useful to build RMHD algorithm by Del Zanna et al. (2003). Finally, a new algorithm to recover the primitive variables from the conserved ones has been implemented (Sect. 2.3.3).

\subsection{Equations of ideal relativistic $M H D$}

The equations that describe the evolution of an ideal relativistic magneto-fluid can be written in the form of conservation laws (see, e.g., Anile 1989, for a full derivation of the equations), the conservation of mass,

$\nabla_{\alpha}\left(\rho u^{\alpha}\right)=0$ 
and the conservation of total energy-momentum,

$\nabla_{\beta} T^{\alpha \beta}=0$,

where $\nabla_{\alpha}$ is the covariant derivative with respect to the metric tensor $g_{\alpha \beta}$. The energy-momentum tensor of a magnetic perfect fluid is given by ${ }^{1}$.

$T^{\alpha \beta}=\rho h^{*} u^{\alpha} u^{\beta}+p^{*} g^{\alpha \beta}-b^{\alpha} b^{\beta}$.

Here $\rho$ is the rest mass density of the fluid, $u^{\alpha}$ the 4-velocity, $b^{\alpha}$ the magnetic field measured by a comoving observer, $h^{*}$ and $p^{*}$ are the hydromagnetic specific enthalpy and the hydromagnetic total pressure, respectively, given by

$h^{*}=h+\frac{b^{\alpha} b_{\alpha}}{\rho}=1+\varepsilon+\frac{p}{\rho}+\frac{|b|^{2}}{\rho} \quad$ and $\quad p^{*}=p+p_{\mathrm{mag}}$,

where $p$ is the fluid pressure, $\varepsilon$ denotes the specific internal energy, $h=1+\varepsilon+p / \rho$ is the specific enthalpy,

$p_{\text {mag }}=\frac{|b|^{2}}{2}$

is the magnetic pressure, and $|b|^{2}=b^{\alpha} b_{\alpha}$ is the magnetic energy density.

Introducing the 3-velocity vector $v^{i}=u^{i} / u^{0}$ (Latin indices run from 1 to 3 , or $x, y, z$ in Cartesian coordinates), the 4-velocity $u^{\alpha}$ can be written as

$u^{\alpha}=W\left(1, v^{1}, v^{2}, v^{3}\right)$,

where $W=1 / \sqrt{1-v^{2}}$ is the Lorentz factor and $v^{2}=v^{i} v_{i}$. Using the infinite conductivity condition, which states that the electric field in the comoving frame must be zero, the magnetic field $b^{\alpha}$ can be written in terms of the magnetic field measured in the laboratory frame, which is given by the 3 -vector $\boldsymbol{B}$, as follows

$b^{0}=W(\boldsymbol{v} \cdot \boldsymbol{B})$,

$b^{i}=\frac{B^{i}}{W}+v^{i} b^{0}$.

In terms of $\boldsymbol{v}$ and $\boldsymbol{B}$, we have $|b|^{2}=B^{2} W^{-2}+(\boldsymbol{v} \cdot \boldsymbol{B})^{2}$.

The evolution of the magnetic field components is described by the homogeneous Maxwell equation,

$\nabla_{\alpha}\left(u^{\alpha} b^{\beta}-u^{\beta} b^{\alpha}\right)=0$,

whose spatial part (in terms of the magnetic field in the laboratory frame) leads to the induction equation

$\frac{\partial \boldsymbol{B}}{\partial t}-\nabla \times(\boldsymbol{v} \times \boldsymbol{B})=0$,

in complete analogy with the Newtonian case.

The time component of Eq. (9) becomes the usual divergence constraint

$\nabla \cdot \boldsymbol{B}=0$,

which has to be fulfilled at all times.

${ }^{1}$ In the following we will use a system of units where the speed of light in vacuum, $c=1$, and a factor of $1 / \sqrt{4 \pi}$ is absorbed into the definition of the magnetic field. Greek subscripts in 4-vectors run from 0 to 3 . We assume a metric signature $(-,+,+,+)$.
Equations (1), (2) and (10), together with the constraint (11) and the equation of state (EOS)

$p=p(\varepsilon, \rho)$

provide the complete set of RMHD equations.

In the following we will use an ideal EOS with a constant adiabatic index, $\gamma$ :

$p=(\gamma-1) \rho \varepsilon$.

To characterize the importance of the magnetic field, it is useful to introduce the magnetization parameter, $\beta$, which is the ratio of magnetic to gas pressure (defined as the reciprocal of the standard $\beta$-parameter of plasma physics):

$\beta \equiv \frac{p_{\text {mag }}}{p}$.

Following Appl \& Camenzind (1988), we introduce a further parameter that will prove useful in parameterizing RMHD flows of astrophysical interest: the ratio of magnetic energy density to that of the rest mass given by

$\sigma \equiv \frac{|b|^{2}}{\rho} \quad\left(=\frac{2 p_{\mathrm{mag}}}{\rho}\right)$.

Then $h^{*}=h+\sigma$, and for $\sigma \gg h$ the Poynting flux $\rho \sigma W^{2} v$ will be much larger than the material energy flux, $\rho h W^{2} v$. Note that we define the parameter $\sigma$ differently from Appl \& Camenzind (1988) or Komissarov (1999b), who defined it to be the inverse ratio. According to our definition both $\beta$ and $\sigma$ become large for large magnetic fields and zero for non-magnetized plasma.

The speed of sound, $c_{\mathrm{s}}$, can then be calculated from (e.g. Landau \& Lifshitz 1966)

$h c_{\mathrm{s}}^{2}=\left.\frac{\partial p}{\partial \rho}\right|_{\varepsilon}+\left.\frac{p}{\rho^{2}} \frac{\partial p}{\partial \varepsilon}\right|_{\rho}$,

i.e.,

$c_{\mathrm{s}}=\sqrt{\frac{\gamma p}{\rho h}}$.

The Alfvén speed, $c_{\mathrm{a}}$, is defined through (Anile 1989)

$c_{\mathrm{a}}^{2}=\frac{|b|^{2}}{\rho h^{*}}=\frac{\sigma}{h+\sigma}$.

\subsection{Primitive and conserved variables}

The RMHD system of partial differential equations in a flat space-time and in Cartesian coordinates can be cast in conservation form as follows

$\partial_{t} \boldsymbol{U}+\partial_{x} \boldsymbol{F}^{x}+\partial_{y} \boldsymbol{F}^{y}+\partial_{z} \boldsymbol{F}^{z}=\mathbf{0}$,

where $\boldsymbol{U}$ is the vector of conserved quantities and $\boldsymbol{F}^{i}$ are the vectors of fluxes. Written in terms of primitive variables,

$\boldsymbol{V}=\left(\rho, v^{x}, v^{y}, v^{z}, p, B^{x}, B^{y}, B^{z}\right)^{T}$,

and using the symbols $D, S$ and $\tau$ to denote the rest mass density, momentum density vector and energy density measured in 
the laboratory frame, respectively, the conserved quantities, $\boldsymbol{U}$, read

$\boldsymbol{U}=\left(\begin{array}{c}D \\ S^{x} \\ S^{y} \\ S^{z} \\ \tau \\ B^{x} \\ B^{y} \\ B^{z}\end{array}\right) \equiv\left(\begin{array}{c}\rho W \\ \rho h^{*} W^{2} v^{x}-b^{0} b^{x} \\ \rho h^{*} W^{2} v^{y}-b^{0} b^{y} \\ \rho h^{*} W^{2} v^{z}-b^{0} b^{z} \\ \rho h^{*} W^{2}-p^{*}-b^{0} b^{0}-\rho W \\ B^{x} \\ B^{y} \\ B^{z}\end{array}\right)$.

The fluxes, $\boldsymbol{F}^{i}$, are then

$$
\boldsymbol{F}^{i}=\left(\begin{array}{c}
\rho W v^{i} \\
\rho h^{*} W^{2} v^{i} v^{x}+p^{*} \delta_{x}^{i}-b^{i} b^{x} \\
\rho h^{*} W^{2} v^{i} v^{y}+p^{*} \delta_{y}^{i}-b^{i} b^{y} \\
\rho h^{*} W^{2} v^{i} v^{z}+p^{*} \delta_{z}^{i}-b^{i} b^{z} \\
\rho h^{*} W^{2} v^{i}-b^{0} b^{i}-\rho W v^{i} \\
v^{i} B^{x}-B^{i} v^{x} \\
v^{i} B^{y}-B^{i} v^{y} \\
v^{i} B^{z}-B^{i} v^{z}
\end{array}\right)
$$

Note that while system (19) consists of eight conservation equations, only seven components of the fluxes, $F^{i}$, are nontrivial. Owing to the antisymmetric character of the induction Eq. (10) the flux of $B^{i}$ in $i$-direction is always zero. For cylindrical coordinates, the equations are given in Appendix A.

\subsubsection{Spectral decomposition}

Our method exploits a directional splitting algorithm to compute the numerical fluxes across cell interfaces, i.e., in each directional sweep the changes of the variables due to fluxes in the orthogonal directions are assumed to be zero. The fluxes along the sweep direction are computed by solving a one dimensional system, e.g., in the $x$-sweep the system reads

$\partial_{t} \boldsymbol{U}+\partial_{x} \boldsymbol{F}^{x}=0$

where $\boldsymbol{U}$ and $\boldsymbol{F}^{x}$ are given by Eqs. (21) and (22), respectively. Note that in the one dimensional system the flux of $B^{x}$ in $x$-direction is zero. Thus the number of independent variables reduces from 8 to 7 . As explained in detail below, the numerical integration of system (23) requires knowledge of the corresponding eigenvalues $\lambda$, which are the solutions of the characteristic polynomial

$\operatorname{det}\left(\mathcal{A}^{x}-\lambda \mathcal{A}^{t}\right)=0$,

where $\mathcal{A}^{x}$ and $\mathcal{A}^{t}$ are Jacobian matrices defined as

$\mathcal{A}_{i j}^{x}=\frac{\partial \boldsymbol{F}_{i}^{x}}{\partial \boldsymbol{V}_{j}} \quad$ and $\quad \mathcal{A}_{i j}^{t}=\frac{\partial \boldsymbol{U}_{i}}{\partial \boldsymbol{V}_{j}}$

respectively. Here $i, j=1 \ldots 7$ denote the seven (independent) components of the vectors (20), (21) and (22).

Equation (24) yields a polynomial of degree seven, the solution of which is non-trivial. Anile (1989) tackled the problem using a fully covariant formulation of the RMHD system. The new system, formed by ten evolution equations instead of seven, leads to the appearance of three additional non-physical waves that have to be discarded. The remaining seven waves are physical. The corresponding eigenvalues can be found in, e.g., Anile (1989). The speeds (eigenvalues) of the four magnetosonic (ms) waves (two slow and two fast ones, sms and fms, respectively) are given by the roots of a quartic polynomial in $\lambda_{\mathrm{ms}}$ (C.1) which does not have a simple analytic solution, i.e., it has to be solved numerically (Sect. 2.3.1).

In RMHD there exist degeneracies where system (23) is not strictly hyperbolic (for an extended discussion see, e.g., Komissarov 1999a). The two possible degenerate cases can be characterized in terms of the components of the magnetic field parallel $\left(b_{\mathrm{p}}\right)$ and normal $\left(b_{\mathrm{n}}\right)$ to the Alfvén-wavefronts in the fluid rest frame. Particularly, when $b_{\mathrm{n}}=0$, the slow magnetosonic and the Alfvén waves propagate at the same speed as the entropy wave, it is possible to find an analytic solution to the eigenvalue problem Eq. (24) taking into account that, applied to the one dimensional system (23), $b_{\mathrm{n}}=0$ in the fluid rest frame implies $B^{x}=0$ in the laboratory frame, i.e., the quartic equation factors into two parts: a quadratic equation in $\lambda$ whose solutions are the two fast magnetosonic waves, and a trivial factor $\left(v^{x}-\lambda\right)^{2}$. The complete set of eigenvalues is hence given by

$\lambda_{0}=v^{x} \quad$ (five times degenerate)

and

$$
\begin{aligned}
\lambda_{\mathrm{fms}}^{ \pm}= & \frac{v^{x}\left(1-\omega^{2}\right)}{1-\boldsymbol{v}^{2} \omega^{2}-R} \\
& \pm \frac{\sqrt{\left(\left(\boldsymbol{v}^{2}-1\right) \omega^{2}+R\right)\left(\left(\boldsymbol{v}^{2}-\left(v^{x}\right)^{2}\right) \omega^{2}+\left(v^{x}\right)^{2}-1+R\right)}}{1-\boldsymbol{v}^{2} \omega^{2}-R}
\end{aligned}
$$

with $R=c_{\mathrm{s}}^{2}(\boldsymbol{v} \cdot \boldsymbol{B})^{2} /\left(\rho h^{*} W^{2}\right)$ and $\omega^{2}=c_{\mathrm{s}}^{2}+c_{\mathrm{a}}^{2}-c_{\mathrm{s}}^{2} c_{\mathrm{a}}^{2}$. In the second degenerate case the characteristic polynomial (24) does not have such a simple solution.

\subsection{Numerical implementation}

In this section, we will describe the main ingredients of our numerical algorithm. We apply a method of lines (e.g., LeVeque 1991) to the equations written in conservation form. This method consists of a spatial discretization step during which the numerical fluxes (Sect. 2.3.1) and the source terms are computed using different types of inter cell reconstructions of the primitive variables (Sect. 2.3.2). Subsequently, the remaining system of semi-discrete ordinary differential equations is integrated in time using a multi-step Runge-Kutta algorithm developed by Shu \& Osher (1988) which provides up to third order accuracy. Our implementation also includes a second order time accurate version of the algorithm. A set of test problems which have been successfully passed by the code are presented in Appendix B.

\subsubsection{Flux formula}

Numerical fluxes across cell interfaces are computed using the HLL flux formula (Harten et al. 1983) similar as in 
Del Zanna et al. (2003) and Gammie et al. (2003). The flux formula is based on the calculation of the maximum speed of perturbations propagating into the states left and right of the cell interface:

$$
\widehat{\boldsymbol{F}}\left(\boldsymbol{U}_{L}, \boldsymbol{U}_{R}\right)=\frac{\psi_{+} \boldsymbol{F}\left(\boldsymbol{U}_{L}\right)-\psi_{-} \boldsymbol{F}\left(\boldsymbol{U}_{R}\right)+\psi_{+} \psi_{-}\left(\boldsymbol{U}_{R}-\boldsymbol{U}_{L}\right)}{\psi_{+}-\psi_{-}},
$$

where

$\psi_{+}=\max \left(0, \lambda_{\mathrm{fms}, L}^{+}, \lambda_{\mathrm{fms}, R}^{+}\right)$,

$\psi_{-}=\min \left(0, \lambda_{\mathrm{fms}, L}^{-}, \lambda_{\mathrm{fms}, R}^{-}\right)$.

These speeds are computed from the characteristic quartic polynomial (C.1, and can effectively be obtained with a Newton-Raphson iteration scheme starting from $\lambda= \pm 1$.

Although they are presently not implemented in our code, we have explored several numerical and analytical methods (including the method described in Abramowitz \& Stegun 1965 and used by Del Zanna et al. 2003) to find the four roots of the quartic Eq. (C.1). According to our tests the best procedure is (1) to compute the two fast magnetosonic wave speeds using a Newton-Raphson iteration scheme; then (2) to reduce the quartic to a quadratic equation by polynomial division; and finally (3) to obtain the two slow magnetosonic wave speeds by a combination of Newton-Raphson iteration and bisection schemes (Press et al. 1992). However, even this best procedure leads to severe numerical problems for high Lorentz factors $(W \gtrsim 40)$.

In the code we use the analytical solution for the left- and right-propagating waves as given by Eq. (27) (corresponding to the first degenerate case) for $\boldsymbol{v} \cdot \boldsymbol{B}=0$. The resulting speeds are always smaller than the speed of light and provide very good (i.e., within 1\%) lower and upper bounds to the actual slowest and fastest magnetosonic wave speeds (as given by the numerical solution of the quartic), respectively. Because of the latter property (proved in Appendix C), the estimates are ideally suited to be used in the HLL flux formula (28). Our analytic estimates are more precise than those reported by Gammie et al. (2003) obtained from the solution of an approximate RMHD dispersion relation. The estimates of the maximum and minimum eigenvalue are also used to compute the Courant time step condition.

The resulting HLL algorithm (as those of Del Zanna et al. 2003; and Gammie et al. 2003) is robust and very efficient computationally. The implementation of more refined Riemann solvers or flux formulas requiring the complete set of eigenvalues and eigenvectors (like, e.g., Balsara 2001; Komissarov 1999b; or Koldoba et al. 2002) is very difficult to realize because of the presence of the RMHD degeneracies discussed above. This may change once a consistent set of eigenvectors is found which can handle both non-degenerate and degenerate states.

\subsubsection{Higher order of accuracy}

In order to increase the spatial order of the accuracy of the code, we have implemented two algorithms for reconstructing variables within numerical cells.
Piecewise linear method (PLM) We use a modified version of the minmod linear interpolation algorithm (e.g., LeVeque 1991) for the one dimensional reconstruction of the variables within cells. If $a$ is one of the variables to be reconstructed and $a_{k}$ is its value in zone $k$, we construct the slopes $\Delta_{k}$ :

$\Delta_{k}=0.5\left(\operatorname{sign}\left(s_{+}\right)+\operatorname{sign}\left(s_{-}\right)\right) \min \left(\left|s_{+}\right|,\left|s_{-}\right|\right)$

where

$s_{+}=\frac{a_{k+1}-a_{k}}{x_{k+1}-x_{k}}, \quad s_{-}=\frac{a_{k}-a_{k-1}}{x_{k}-x_{k-1}}$.

Values of $a$ at the left and right interface of the zone, $a_{L, k}$ and $a_{R, k}$, are then computed according to

$a_{L, k}=a_{k}+\Delta_{k}\left(x_{k-1 / 2}-x_{k}\right)$,

$a_{R, k}=a_{k}+\Delta_{k}\left(x_{k+1 / 2}-x_{k}\right)$.

The monotonicity of the data is preserved when taking the minimum of $\left|s_{+}\right|$and $\left|s_{-}\right|$at every numerical cell, whereas the TVD property is accomplished by switching to first order (i.e., piecewise constant data) at local extrema (where $\Delta_{k}=0$ ).

In the $x$ sweep the reconstruction procedure just described is applied to the variables $\left\{\ln \rho, \ln p, W v^{x}, W v^{y}, W v^{z}, B^{y}, B^{z}\right\}$. Interpolating the logarithms of density and pressure reduces the magnitude of the jumps at cell interfaces with large gradients of density and/or pressure. Finally, and here is where the modification with respect to the standard minmod algorithm takes place, we limit the absolute value of the slope to 2 when interpolating $\ln \rho$ and $\ln p$ in those zones where the magnetization parameter $\beta$ (14) is larger than a certain threshold (between 4 and 10 in our applications). Reconstruction of $B^{x}$ is unnecessary in the $x$ sweep, as the magnetic field is originally defined on a staggered grid (see Sect. 2.3.4). In the $y$ and $z$ sweep the primitive variables are reconstructed in a similar way, but permuting the indices $(x, y, z)$ cyclically.

Piecewise parabolic method (PPM). From the original relativistic version of the PPM algorithm of Martí \& Müller (1996) we only adopt the cell reconstruction in order to construct monotonic parabola. For each zone $k$ the quartic polynomial is obtained, which has zone-averaged values $a_{k-2}, a_{k-1}, a_{k}, a_{k+1}$ and $a_{k+2}$, where $a$ is the variable to be reconstructed (the same as in the PLM algorithm). The polynomial interpolates the structure inside the zone, and provides the values at the left and right interface of the zone, $a_{L, k}$ and $a_{R, k}$. These reconstructed values are then modified such that the parabolic profile defined by $a_{L, k}, a_{R, k}$ and $a_{k}$ is monotonic inside the zone. The modified interpolated values at the zone interfaces define local Riemann problems which are solved by Eq. (28). Near contact discontinuities the interpolation procedure is slightly modified to produce narrower jumps. In the vicinity of shocks the scheme switches (locally) to a piecewise constant approximation in order to avoid spurious post shock oscillations (Appendix I in Martí \& Müller 1996). The original relativistic hydrodynamic algorithms for the detection of contact discontinuities and shocks can also be used in RMHD provided the character of the discontinuity does not change in the presence of a magnetic field: in a shock there is a jump of the thermal pressure 
accompanied by a negative value of the divergence of the velocity field and, in a contact discontinuity there is a jump in density without change of normal velocity.

\subsubsection{Recovery of primitive variables}

The code evolves the conserved quantities $\{D, S, \tau, \boldsymbol{B}\}$, but not the primitive variables $\{\rho, \boldsymbol{v}, p, \boldsymbol{B}\}$. Therefore, an algorithm is required which computes the latter from the zone-averaged values of the former set. Since the conserved quantities can be written in terms of the primitives in an analytic closed form, but not the other way around, one has to employ iterative algorithms to compute the primitive variables.

We use Eqs. (21) and (13) to construct two functions $\mathcal{F}_{1}$ and $\mathcal{F}_{2}$ depending on the Lorentz factor $W$ and the variable $Z \equiv \rho h W^{2}$ (the inertial rest mass density for an ideal gas),

$$
\begin{aligned}
\mathcal{F}_{1}(Z, W)= & \frac{\tau+D}{1+\boldsymbol{B}^{2}}-\frac{Z+\boldsymbol{B}^{2}}{1+\boldsymbol{B}^{2}}+\frac{(\boldsymbol{B} \cdot \boldsymbol{S})^{2}}{2 Z^{2}\left(1+\boldsymbol{B}^{2}\right)} \\
& +\frac{\gamma-1}{\gamma} \frac{Z-D W}{W^{2}\left(1+\boldsymbol{B}^{2}\right)}+\frac{\boldsymbol{B}^{2}}{2 W^{2}\left(1+\boldsymbol{B}^{2}\right)}
\end{aligned}
$$

and

$$
\begin{aligned}
\mathcal{F}_{2}(Z, W)= & 1-\frac{1}{W^{2}}-\frac{1}{\left(Z+\boldsymbol{B}^{2}\right)^{2}}\left(\boldsymbol{S}^{2}+2 \frac{(\boldsymbol{B} \cdot \boldsymbol{S})^{2}}{Z}\right. \\
& \left.+\boldsymbol{B}^{2}\left(\frac{\boldsymbol{B} \cdot \boldsymbol{S}}{Z}\right)^{2}\right) .
\end{aligned}
$$

The common zero of both functions - computed with a two dimensional Newton-Raphson iteration (function mnewt in Press et al. 1992) - yields values for $Z$ and $W$, which are used to calculate the primitive variables via

$v^{i}=\frac{S^{i}+\frac{b^{0}}{W} B^{i}}{Z+\boldsymbol{B}^{2}} \quad$ where $\quad \frac{b^{0}}{W}=\frac{\boldsymbol{B} \cdot \boldsymbol{S}}{Z}$,

$\rho=\frac{D}{W} \quad$ and $\quad p=\frac{\gamma-1}{\gamma} \frac{Z-D W}{W^{2}}$.

The internal energy $\varepsilon$ follows then from Eq. (13).

This recovery scheme yields physical results for a wide range of parameters. Using in FORTRAN double-precision arithmetics, for an initial guess which differs from the solution by less than about $20 \%$, a tolerance of $10^{-8}$, and magnetic fields of the order of $10^{-5}$, the recovery provides physical solutions (expressed in code units) for $10^{-10}<p<10^{8}$, $10^{-9}<\rho<10^{8}$, and $1<W<10^{4}$. For stronger fields these intervals get smaller, while for smaller fields they become larger. If no magnetic field is present, the recovery works for density and pressure values as low as $10^{-17}$ up to $W=10^{4}$. It also works for strong magnetic fields, if $\beta<1$, or in general if the magnetic energy density and the Poynting flux do not much exceed the internal plus kinetic energy density and the hydrodynamic momentum fluxes, respectively. The parameter range where the recovery algorithm works properly widens when better initial values are available for the iterative procedure.

\subsubsection{Conservation of $\nabla \cdot B=0$}

The equations for the rest mass, momentum and energy are integrated using the numerical fluxes given in Eq. (28), while the equations for the magnetic field are evolved in a different way in order to keep $\nabla \cdot \boldsymbol{B}=0$ during the simulation. The latter constraint is not trivially fulfilled although it is implicitly encoded in the RMHD Eqs. (19). To guarantee the constraint also numerically, we use the constrained transport (CT) method developed by Evans \& Hawley (1988) and adapted to Godunovtype schemes by Ryu et al. (1998), which keeps $\nabla \cdot \boldsymbol{B}=0$ up to machine precision, but which has the disadvantage that it is at most second order accurate in space (see, Londrillo \& Del Zanna (2004) for higher order implementations of the divergence free condition).

We define two sets of magnetic field vectors (for the sake of clarity we will restrict ourselves here to the algorithm for two dimensional problems):

1. the zone centered vector $\widetilde{\boldsymbol{B}}_{k, l}$ defined at coordinates $\left(x_{k}, y_{l}\right)$; and

2. the staggered interface magnetic field $\widehat{\boldsymbol{B}}_{k, l}$, where $\widehat{B}_{k, l}^{x}$ is defined at $\left(x_{k-1 / 2}, y_{l}\right)$ and $\widehat{B}_{k, l}^{y}$ at $\left(x_{k}, y_{l-1 / 2}\right)$.

Note that we introduced a slight inconsistency in the notation in order to avoid formulae cluttered with indices.

The zone centered vector $\widetilde{\boldsymbol{B}}$, which is required for setting the boundary conditions, and for calculating the source terms and the fluxes of the other variables, is computed in every sweep by a simple interpolation from the staggered field components

$\widetilde{B}_{k, l}^{x}=\frac{1}{2}\left(\widehat{B}_{k, l}^{x}+\widehat{B}_{k+1, l}^{x}\right)$.

and

$\widetilde{B}_{k, l}^{y}=\frac{1}{2}\left(\widehat{B}_{k, l}^{y}+\widehat{B}_{k, l+1}^{y}\right)$.

The field components are updated by applying the Runge-Kutta time integration algorithm to the set of equations

$\frac{\mathrm{d} \widehat{B}_{k, l}^{x}}{\mathrm{~d} t}=-\frac{1}{\Delta y}\left(\Omega_{k, l+1}-\Omega_{k, l}\right)$

and

$\frac{\mathrm{d} \widehat{B}_{k, l}^{y}}{\mathrm{~d} t}=+\frac{1}{\Delta x}\left(\Omega_{k+1, l}-\Omega_{k, l}\right)$,

where

$$
\begin{aligned}
\Omega_{k, l}= & \frac{1}{2}\left(\widehat{F}^{y}\left(B^{x}\right)_{k-1, l}+\widehat{F}^{y}\left(B^{x}\right)_{k, l}\right. \\
& \left.-\widehat{F}^{x}\left(B^{y}\right)_{k, l-1}-\widehat{F}^{x}\left(B^{y}\right)_{k, l}\right)
\end{aligned}
$$

corresponds to the $z$-component of $\boldsymbol{B} \times \boldsymbol{v}$ computed at the lower left corner of the zone centered at $\left(x_{k}, y_{l}\right)$, while $\widehat{F}^{y}\left(B^{x}\right)_{k, l}$ and $\widehat{F}^{x}\left(B^{y}\right)_{k, l}$ are the numerical advective fluxes in $y$ and $x$-direction across the interface at $\left(x_{k-1 / 2}, y_{l}\right)$ and $\left(x_{k}, y_{l-1 / 2}\right)$ corresponding to $B^{x}$ and $B^{y}$, respectively, i.e.,

$$
\begin{aligned}
\widehat{F}^{y}\left(B^{x}\right)_{k, l} & =\left(\widehat{v^{y} B^{x}}\right)_{k, l} \\
\widehat{F}^{x}\left(B^{y}\right)_{k, l} & =\left(\widehat{v^{x} B^{y}}\right)_{k, l} .
\end{aligned}
$$


It is easy to show that Eqs. (40)-(42) conserve the following discretized version of the divergence of the magnetic field:

$(\nabla \cdot \boldsymbol{B})_{k, l} \equiv \frac{\widehat{B}_{k+1, j}^{x}-\widehat{B}_{k, l}^{x}}{\Delta x}+\frac{\widehat{B}_{k, l+1}^{y}-\widehat{B}_{k, l}^{y}}{\Delta y}$.

\subsubsection{Boundary conditions}

At the boundaries the computational grid is extended by up to four so-called ghost zones which serve to enforce the boundary conditions for the primitive variables before the spatial interpolation is performed. The number of ghost zones employed in this process depends on the order of the spatial interpolation algorithm (one for PLM, four in case for our implementation of PPM).

A second boundary routine is called after the dimensional sweeps to set the boundary values for the fluxes associated to the magnetic field components. These fluxes only need to be fixed in the first ghost zone (see Eq. (42)), e.g., when computing $\Omega_{1,1}$ one has to prescribe both $\widehat{F}^{y}\left(B^{x}\right)_{0,1}$ and $\widehat{F}^{x}\left(B^{y}\right)_{1,0}$. Usually fluxes at the ghost zone are set in such a way that zero flux gradients are enforced.

\section{Simulations}

\subsection{Simulation setup}

\subsubsection{The numerical grid}

All jet simulations in this work are performed on a $2 \mathrm{D}$ equidistant grid in cylindrical coordinates, $(r, z)$, assuming axisymmetry. The simulations themselves are 2.5-dimensional as we evolve all three spatial components of vectors using Eqs. (A.2)-(A.9), and thus include a toroidal magnetic field and flow velocity.

The simulated jets are produced by injecting beam matter into the grid along the $z$ axis through a nozzle of radius $r_{\mathrm{b}}$ (the beam radius) at $z=0$. Outside the nozzle, i.e., for $r>r_{\mathrm{b}}$ and $z=0$ we impose special reflecting boundary conditions assuming that the axial and toroidal velocity and field components are mirrored. This is justified by the presence of a twin counterjet in actual radio galaxies, and eliminates the Lorentz force in the equatorial plane. The assumed axisymmetry implies reflecting boundary conditions on the symmetry axis at $r=0$, where the radial and toroidal velocity and magnetic field components are mirrored, i.e., they are zero at zero radius. At the two outer edges of the grid at $r=r_{\max }$ and $z=z_{\max }$, respectively, we impose zero gradient boundary conditions. Material is allowed to freely leave the grid there.

Initially the computational domain is filled with a uniform medium at rest having the same thermal pressure and adiabatic index as the jet. The initial magnetic field configuration depends on the type of simulation.

\subsubsection{Jet parameterization}

Every jet simulation is fully specified by setting the following independent parameters:

- the flow speed of the beam at the nozzle, $v_{\mathrm{b}}$ (associated Lorentz factor, $W_{\mathrm{b}}$ );
- the ratio of the rest mass densities of the beam fluid and the external medium, $\eta$;

- the adiabatic index, $\gamma$, used in the equation of state (13);

- the internal (classical) Mach number of the beam at the nozzle $M_{\mathrm{b}}=v_{\mathrm{b}} / c_{\mathrm{s}, \mathrm{b}}$ (which controls the thermodynamic relativistic effects of the model) or its relativistic analogue, the proper Mach number (Königl 1980), $\mathcal{M}_{\mathrm{b}}=M_{\mathrm{b}} W_{\mathrm{b}} / W_{\mathrm{s}, \mathrm{b}}$, where $W_{\mathrm{s}, \mathrm{b}} \equiv\left(1-c_{\mathrm{s}, \mathrm{b}}^{2}\right)^{-1 / 2}$ is the Lorentz factor associated with the local sound speed in the beam;

- the magnetization parameter, $\beta$ (Eq. (14)), both of the beam fluid and the external medium.

The other parameter, $\sigma$, defined by Eq. (15), will prove useful when discussing the simulations. However, as it is not independent of the other parameters, it need not to be specified for a jet model.

We point out here that there are three ways for a magnetized jet to be relativistic. First, when the flow velocity is close to $c$, and therefore the Lorentz factor of the flow is much larger than one. Second, when the beam plasma is hot, i.e., $\varepsilon_{\mathrm{b}} \gg c^{2}$ such that $h_{\mathrm{b}} \gg 1$ and the sound speed (17) are relativistic approaching the maximum value $\sqrt{\gamma-1}(\approx 0.58 c$ for $\gamma=4 / 3$, and $\approx 0.83 c$ for $\gamma=5 / 3$ ). In this case, the internal beam Mach number, $M_{\mathrm{b}}$, approaches the minimum value for a given $v_{\mathrm{b}}$ $\left(1.73 v_{\mathrm{b}}\right.$ for $\gamma=4 / 3$, and $1.22 v_{\mathrm{b}}$ for $\left.\gamma=5 / 3\right)$. Finally, when $\sigma_{\mathrm{b}} \gg 1$ such that $h_{\mathrm{b}}^{*} \gg 1$, and when $h_{\mathrm{b}}^{*} \gg h_{\mathrm{b}}$, the Alfvén speed (18) is close to $c$.

\subsubsection{Magnetization parameter}

When simulating a jet with a non-uniform magnetic field, the magnetization parameter of the beam is given by the average value, $\bar{\beta}$, across the beam. In our simulations of jets with purely toroidal magnetic fields presented below we have assumed the same profile for the magnetic field as Lind et al. (1989) and Komissarov (1999b), corresponding to a beam in transverse hydromagnetic equilibrium with a core of uniform electric current with radius $r_{\mathrm{m}}$ - the magnetization radius - and a shell without any current. The (toroidal) magnetic field in the beam, $b_{\mathrm{b}}^{\phi}$, is then given by

$b_{\mathrm{b}}^{\phi}=\left\{\begin{array}{lll}b_{\mathrm{m}} \frac{r}{r_{\mathrm{m}}} & \text { if } & r<r_{\mathrm{m}}, \\ b_{\mathrm{m}} \frac{r_{\mathrm{m}}}{r} & \text { if } & r \geq r_{\mathrm{m}}, \\ 0 & \text { if } & r>r_{\mathrm{b}},\end{array}\right.$

where $b_{\mathrm{m}}$ is the magnetic field strength at the magnetization radius $r_{\mathrm{m}}$.

Averaging (44) over the beam cross section at the nozzle and dividing by the uniform thermal beam pressure, $p_{\mathrm{b}}$, yields

$\bar{\beta}=\frac{b_{\mathrm{m}}^{2} r_{\mathrm{m}}^{2}\left(0.25-\ln \left(r_{\mathrm{m}} / r_{\mathrm{b}}\right)\right)}{p_{\mathrm{b}} r_{\mathrm{b}}^{2}}$.

From $p_{\mathrm{b}}, \bar{\beta}$ and $r_{\mathrm{m}}$ one obtains $b_{\mathrm{m}}$ through (45). Finally, the toroidal magnetic field in the beam as seen in the laboratory frame is $B_{\mathrm{b}}^{\phi}=W_{\mathrm{b}} b_{\mathrm{b}}^{\phi}$. 
In the simulations with purely poloidal magnetic fields we have chosen a simpler initial setup. The grid is initially filled with a uniform axial magnetic field, $B_{\mathrm{a}}^{z}$, which according to the definitions of $|\boldsymbol{b}|^{2}$ and $\beta$ is given by

$B_{\mathrm{a}}^{z}=\sqrt{2 \beta p_{\mathrm{b}}}$.

Through the nozzle the same field is injected with the flow, i.e., $B_{\mathrm{b}}^{z}=B_{\mathrm{a}}^{z}$.

\subsubsection{Analysis tools}

Besides the analysis and comparison of the distributions of restmass density, flow velocity, magnetic field lines, etc. for the different models, we introduce a number of global quantities that are used to characterize some morphodynamical aspects of the models. These quantities are:

- the head position of the jet is the $z$-coordinate of the leading bow shock, and hence the length of the cavity, $l_{\mathrm{c}}$;

- the associated jet velocity, $v_{\mathrm{h}}$, i.e., the change of the length of the cavity as a function of time;

- the (mean) velocity of the terminal (Mach) shock, $v_{\mathrm{M}}$;

- the cylindrical aspect ratio, $A_{\mathrm{c}} \equiv l_{\mathrm{c}} / r_{\mathrm{c}}$, is the aspect ratio of a cylinder which has the same volume and length, $l_{\mathrm{c}}$, as the cavity, i.e., $r_{\mathrm{c}}=\sqrt{V_{\mathrm{c}} /\left(\pi l_{\mathrm{c}}\right)}$;

- various mean values along the axial direction defined as $\langle A\rangle=\sum_{z_{k}=1}^{z_{k}=n_{z}} A\left(r_{i}, z_{k}\right) / n_{z}$, where $A$ is a physical quantity whose zone average value is $A\left(r_{i}, z_{k}\right)$ in zone $\left(r_{i}, z_{k}\right)$.

As in Aloy et al. (1999a, 2000); Aloy et al. (2003), the code also evolves a variable, $f$, representing the beam mass fraction. In order to trace the beam material across the computational domain, this tracer variable is set to $f=1$ for the material injected through the nozzle, and to $f=0$ for the ambient medium. In the following, the beam mass fraction will be used to identify different parts of the jet: zones where $f \geq 0.9$ belong to the beam, and zones where $0.1<f<0.9$ belong to the cocoon. The cavity is defined as the region where $f>0$. Note that cavity and cocoon are different structures according to these definitions, whereas in the literature the terms cocoon and cavity are sometimes used interchangeably.

A one dimensional estimate for the head advance speed of a non-magnetized, relativistic jet can be obtained by equating the momentum flux of the beam and the ambient gas in the frame of the working surface (Martí et al. 1997):

$v_{\mathrm{h}}^{1 D}=\frac{\sqrt{\eta_{R}^{*}}}{1+\sqrt{\eta_{R}^{*}}} v_{\mathrm{b}}$

with $\eta_{R}^{*}=\eta_{R} W_{\mathrm{b}}^{2}$, where $\eta_{R}=\left(\rho_{\mathrm{b}} h_{\mathrm{b}}\right) /\left(\rho_{\mathrm{a}} h_{\mathrm{a}}\right)$ is the ratio of the enthalpies of the beam and the ambient medium (subscript a denotes the ambient medium). Even though this expression does not hold for magnetized jets, it will prove to be a very good estimate, as discussed below.

The evolution of 2D jets can be divided into two phases (Scheck et al. 2002): (1) a 1D phase where the velocity is constant and approximated by (47); and (2) a 2D phase where $2 \mathrm{D}$ effects become important and the jet decelerates. The 2D phase begins when the first major vortex shedding occurs at the terminal Mach disk.
Table 1. Overview of the simulated models: the columns give from left to right the name of the model, the type of the initial field configuration, the adiabatic index $\gamma$, the beam speed $v_{\mathrm{b}}$, the proper beam Mach number $\mathcal{M}_{\mathrm{b}}$, the average magnetization parameter $\bar{\beta}$, and the ratio of the magnetic energy density and the rest mass density $\sigma$.

\begin{tabular}{llrrrrr}
\hline \hline Model & Field & $\gamma$ & $v_{\mathrm{b}}$ & $\mathcal{M}_{\mathrm{b}}$ & $\bar{\beta}$ & $\sigma$ \\
\hline $\mathrm{C} 2-0$ & none & $5 / 3$ & 0.99 & 41.95 & 0 & 0 \\
$\mathrm{C} 2-1 / 20$ & tor & $5 / 3$ & 0.99 & 41.95 & $1 / 20$ & 0.0017 \\
$\mathrm{C} 2-1$ & tor & $5 / 3$ & 0.99 & 41.95 & 1 & 0.0341 \\
$\mathrm{C} 2-10 / 3$ & tor & $5 / 3$ & 0.99 & 41.95 & $10 / 3$ & 0.1135 \\
$\mathrm{C} 2-$ pol-1 & pol & $5 / 3$ & 0.99 & 41.95 & 1 & 0.0341 \\
\hline $\mathrm{B} 1-0$ & none & $4 / 3$ & 0.99 & 41.95 & 0 & 0 \\
$\mathrm{~B} 1-1 / 20$ & tor & $4 / 3$ & 0.99 & 41.95 & $1 / 20$ & 0.0022 \\
$\mathrm{~B} 1-1$ & tor & $4 / 3$ & 0.99 & 41.95 & 1 & 0.0445 \\
$\mathrm{~B} 1-10 / 3$ & tor & $4 / 3$ & 0.99 & 41.95 & $10 / 3$ & 0.1482 \\
$\mathrm{~B} 1-$ pol-1 & pol & $4 / 3$ & 0.99 & 41.95 & 1 & 0.0445 \\
\hline $\mathrm{C} 1-0$ & none & $5 / 3$ & 0.9 & 13.61 & 0 & 0 \\
$\mathrm{C} 1-1$ & tor & $5 / 3$ & 0.9 & 13.61 & 1 & 0.0279 \\
$\mathrm{C} 1-10 / 3$ & tor & $5 / 3$ & 0.9 & 13.61 & $10 / 3$ & 0.0931 \\
$\mathrm{C} 1-$ pol-1 & pol & $5 / 3$ & 0.9 & 13.61 & 1 & 0.0279 \\
\hline
\end{tabular}

\subsection{Model parameters}

We have selected three of the jet models of Martí et al. (1997) to study the effects of magnetic fields on the dynamics and morphology of relativistic jets. All three jets are light $(\eta=0.01)$, supersonic, cold $\left(M_{\mathrm{b}}=6\right)$, and the gas pressure is matched with that of the ambient medium. In the toroidal field models the magnetization radius is $r_{\mathrm{m}}=0.6 r_{\mathrm{b}}$, and the magnetic field (44) is injected with the beam into a uniform, non-magnetized medium. In the poloidal field simulations, the whole grid is filled with a uniform, purely axial magnetic field of the same strength as that injected with the beam according to Eq. (46). The other parameters of the external medium are identical to those of the toroidal field models. The simulations are performed on a uniform grid of $\left(n_{r} \times n_{z}\right)=(420 \times 2000)$ zones covering a domain of $10.5 \times 50$ beam radii, i.e., the resolution is 40 zones $/ r_{\mathrm{b}}$. We point out that this numerical resolution is equivalent to that of Martí et al. (1997), who used a third order algorithm (but only 20 zones $/ r_{\mathrm{b}}$ ), while we only use a second order accurate one. Thus, as in Martí et al. (1997), the most prominent supersonic structures are properly resolved. Of course, even better numerical resolution would be required to study problems like the mass entrainment in the jet or the mixing of beam and ambient plasma in the cavity of the generated jets. The purely hydrodynamic and the toroidal field models are simulated with PLM spatial interpolation, while the poloidal field runs employ PPM interpolation (although in both cases the two spatial interpolations could have been used indistinctly; see Appendix B). All models are evolved until the head of the jet reaches the opposite edge of the computational domain.

For our models (see Table 1) we use the same model names as Martí et al. (1997) extended by suffixes describing the magnetic field strength. For example, the toroidal field model of series $\mathrm{C} 2$ with an average magnetization of 1 (i.e., the equipartition model) is called $\mathrm{C} 2-1$, and the corresponding poloidal 


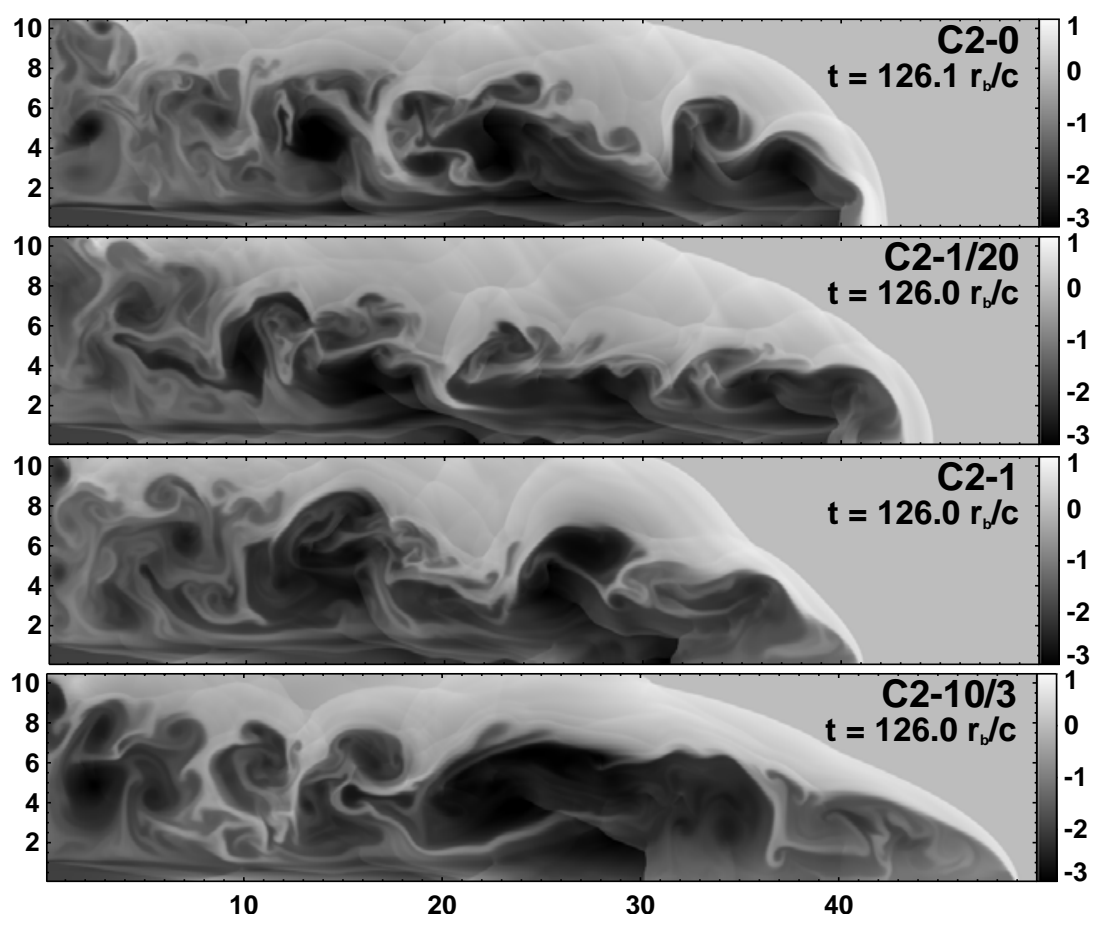

Fig. 1. Snapshots of the logarithm of the rest mass density of models $\mathrm{C} 2-0, \mathrm{C} 2-1 / 20, \mathrm{C} 2-1$ and $\mathrm{C} 2-10 / 3$ at $t \sim 126 \mathrm{r}_{\mathrm{b}} / \mathrm{c}$.

field model is called C2-pol-1. The model corresponding to the pure hydrodynamic case is named $\mathrm{C} 2-0$. All models have $\sigma<1$, i.e., they have a low Poynting flux. The models of series $\mathrm{B} 1$ and $\mathrm{C} 2$ only differ in the adiabatic index $\gamma$, and those of series $\mathrm{C} 1$ and $\mathrm{C} 2$ only in the beam velocity $v_{\mathrm{b}}$. The toroidal velocity at injection is zero in all models, which together with the chosen initial magnetic field configurations prevent the models from generating additional components of magnetic field and flow velocity in the course of evolution.

Our boundary condition at $z=0$ (reflecting outside the nozzle, see previous section) differs from that of Martí et al. (1997) (zero gradients outside the jet nozzle), as does the Riemann solver and the reconstruction procedure. Therefore, the results of our purely hydrodynamic reference simulations are slightly different from their results. We choose the reflecting boundary conditions in order to avoid that a large part of the energy contained in the back flow leaves the grid. This boundary condition is also consistent with that of more recent jet simulations, like e.g., Scheck et al. (2002) and Krause (2003).

Besides the field topology the setup of the toroidal and the poloidal field models differs in other aspects, too. In the toroidal field models $B^{\phi}$ is injected together with the beam flow into a domain filled with a non-magnetized medium. In the poloidal case, however, the computational domain initially already contains a magnetic field of the same strength as that injected with the beam. Hence, in the poloidal field models both the total pressure $p^{*}$ (as in the toroidal field models) and the thermal pressure $p$ are matched at the beam surface, $p$ being uniform and the same inside the beam and in the ambient medium. In contrast, the toroidal field models have a negative radial gradient in $p^{*}$, which is compensated by the magnetic tension.
All jet simulations were carried out on an IBM p690 Regatta computer and required between 8 and $30 \mathrm{~h}$ on a shared memory node with 32 Power4 $1300 \mathrm{MHz}$ processors.

\section{Results}

The most remarkable result we find is that different from classical MHD simulations (e.g., Lind et al. 1989) the inclusion of a magnetic field leads to diverse effects which do not always scale linearly with the relative strength of the field. There are, however, some clear trends which we will discuss in detail below.

\subsection{Series $C 2$}

\subsubsection{C2-0 to C2-10/3: Purely hydrodynamic and toroidal field models}

Figures 1 and 2 show the rest mass density and the Lorentz factor of the purely hydrodynamic model $\mathrm{C} 2-0$, and those of the toroidal field models $\mathrm{C} 2-1 / 20, \mathrm{C} 2-1$ and $\mathrm{C} 2-10 / 3$ (from top to bottom), respectively. Apart from differences in details, one morphological feature is particularly noticeable: with growing toroidal field strength, the supersonic part of the beam ends further behind the leading edge of the bow shock (note the steep decline of the Lorentz factor near the head of the jet in Fig. 2), i.e., the jet becomes transonic much closer to the origin. The high density and pressure structure between the termination of the supersonic beam at the Mach disk and the head of the jet is called nose cone (Clarke et al. 1986) or plug (Lind et al. 1989) (e.g., the region around the axis between 28 and 46 beam radii in the bottom panel of Fig. 1). The nose cone is made up of beam material that it is not deflected backwards into the 


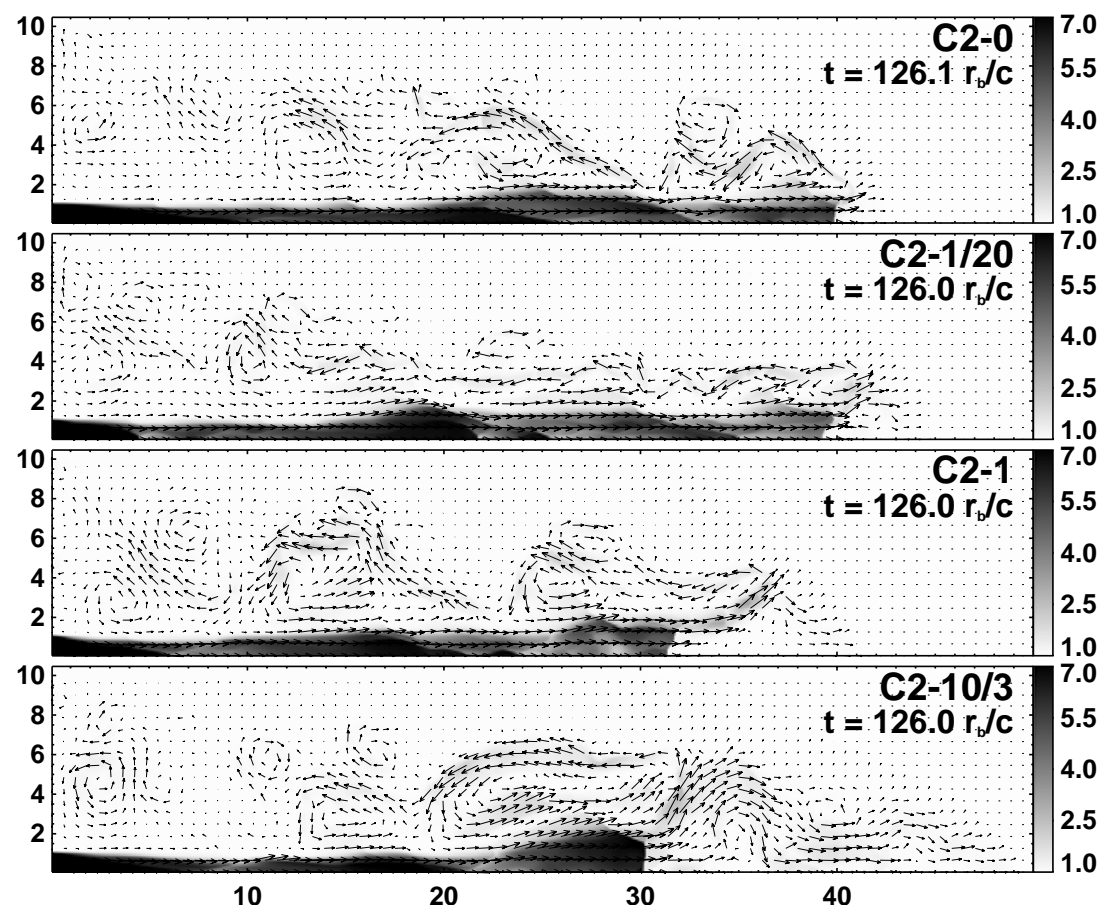

Fig. 2. Same as Fig. 1 but showing snapshots of the Lorentz factor and the flow field.

cocoon, and of entrained ambient material near its rear end. Both the density and the pressure in the nose cone are very high, and show indications of turbulence. Moreover, the pressure in the nose cone is relatively smooth and particularly high on the axis (due to magnetic confinement) where it can reach values $\sim 10^{3}$ times larger than that of the external medium. The nose cone makes the head of the jet appear narrower for larger values of $\bar{\beta}$.

In the cocoon, close to the head of the jet, a number of organized structures appear as a result of the dynamic effects of the toroidal field. The magnetic field confines and suppresses the development of Kelvin-Helmholtz instabilities at the interface cocoon/shocked ambient medium, and the material deflected into the cocoon after passing the Mach disk starts to fill a low density bubble. With increasing toroidal field strength the bubble grows larger in size. In model C2-10/3 it roughly stretches from $z=20 r_{\mathrm{b}}$ up to $z=35 r_{\mathrm{b}}$, and is confined by a shell of highly magnetized material (Fig. 3). The Mach disk and the terminal shock of the beam reside in the middle of the bubble. The beam flow is terminated at the Mach disk, and matter has to flow around it to reach the head of the jet (Fig. 2). In model $\mathrm{C} 2-1$ the shocked flow around the Mach disk reaches relativistic speeds with Lorentz factors of 3 and more. The average beam Lorentz factor decreases with increasing magnetization of the beam.

In models $\mathrm{C} 2-1$ and $\mathrm{C} 2-10 / 3$ the magnetization $\beta$ (Fig. 3) decreases drastically across the first cross shock due to an increase in the thermal pressure (Fig. 4). It remains low inside the beam up to the terminal shock, increases downstream of this shock, and becomes even larger when the shocked plasma begins to flow away radially from the jet axis. The pinching force provided by the toroidal field in that region suppresses any further sideways expansion of the jet, and pushes a large fraction of the plasma into the nose cone. In model $\mathrm{C} 2-10 / 3$ this pinching is strongest at $z=38 r_{\mathrm{b}}$ (see the bottom panel of Fig. 2). The nose cone contains primarily highly magnetized plasma which leads to a strong pinching force confining the flow close to the axis. The magnetic field accumulates at the inside of the high density shell (the shroud of Lind et al. 1989) of the jet, where the thermal pressure is low and $\beta$ is large (Fig. 3). This magnetized shell will influence the emission properties of the radio lobes in actual sources, as it contains the largest and most ordered return current (Fig. 4, top panel), which occurs because there is no confining field in the ambient medium, and because this environment behaves as a perfect conductor (Begelmann et al. 1984). However, the structure of the return current becomes highly anisotropic throughout the cocoon and at the surface of the jet. The magnetic field itself is largest in the beam and in the nose cone (see the two bottom panels of Fig. 3).

\subsubsection{C2-pol-1: Poloidal field model}

Figure 5 shows the rest mass density, the Lorentz factor with the flow field, the logarithm of the modulus of the magnetic field with the field lines superimposed, and the magnetization parameter $\beta$ for the poloidal field model C2-pol-1. Although the overall morphology and structure of the jet and the cocoon of this model are very similar to those of other models of series $\mathrm{C} 2$, there are a number of striking differences. In particular, we will compare model $\mathrm{C} 2-$ pol-1 both with the hydrodynamic model $\mathrm{C} 2-0$ and the toroidal field model having the same magnetization $(\mathrm{C} 2-1)$.

First of all, instead of forming a narrow nose cone structure, the beam broadens close to its termination point giving the head of the jet a hammer-like appearance caused by the emission of vortices from the head of the jet. The beam plasma flows along 


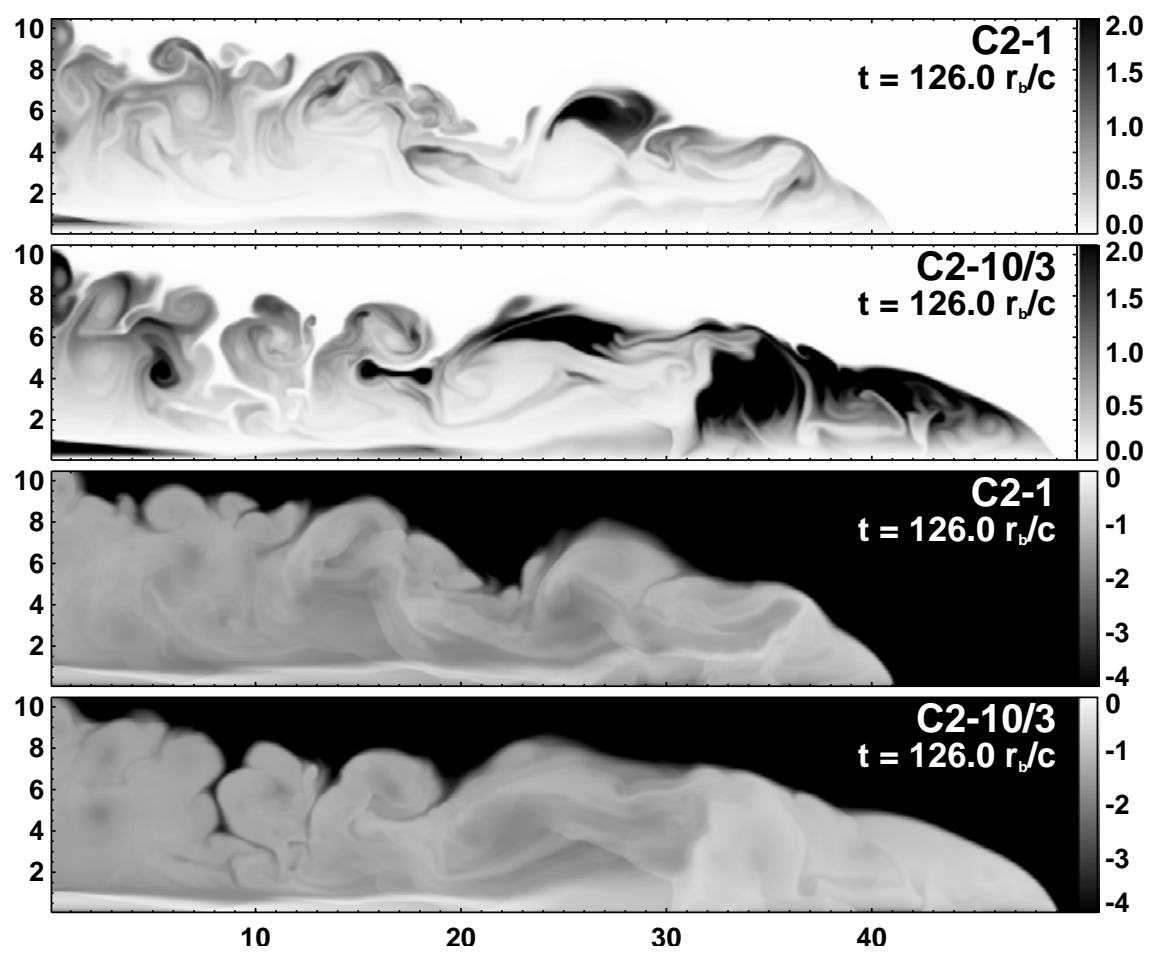

Fig. 3. Snapshots of the magnetization parameter $\beta$ (upper two panels) and the toroidal field component $B^{\phi}$ (lower two panels) of the two most strongly magnetized models of series $\mathrm{C} 2$ at $t \sim 126 r_{\mathrm{b}} / c$.

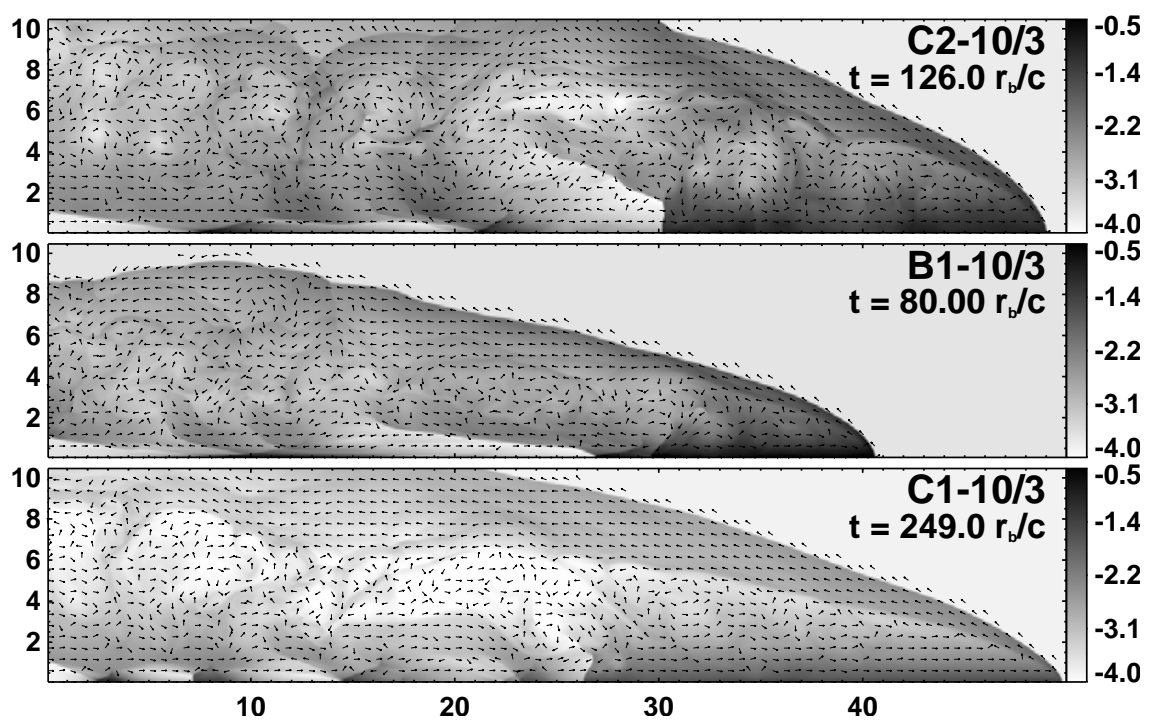

Fig. 4. Snapshots of the logarithm of the pressure for all the models having a toroidal field with $\bar{\beta}=10 / 3$. The overlaid vector field shows the structure of the currents $(\nabla \times B /|\nabla \times B|)$.

the field lines up to the head of the jet, where it is deflected to form a cocoon (second panel of Fig. 5) dragging along the magnetic lines.

Model C2-pol-1 also differs from all the other models in the strength of the cross shocks on the axis. While the first cross shock in model $\mathrm{C} 2-$ pol-1 occurs much nearer to the nozzle (at $z=8 r_{\mathrm{b}}$ ) than in model $\mathrm{C} 2-0$, it is also much weaker (the density contrast is $\sim 8$ and $\sim 3$ times smaller than in models C2-10/3 and C2-1, respectively) and has the least extended post-shock region (in axial direction) of all models. Downstream of this shock, the beam pressure and density remain low (below $\sim 5 p_{\mathrm{b}}$ ) until the flow reaches the second cross shock, which is weak compared to those of the other models, too. It is especially weak compared to the toroidal field model with the same average $\beta$. This behavior results from the increase of the radially (outward) directed magnetic tension force which straightens the poloidal field lines, which are bent towards the axis due to external radial pressure forces and thereby repel waves driven into the beam. Thus, during the whole evolution the beam remains much less affected by shocks than in the models with toroidal magnetic fields. The terminal shock, however, is as strong as in model $\mathrm{C} 2-1$ or 


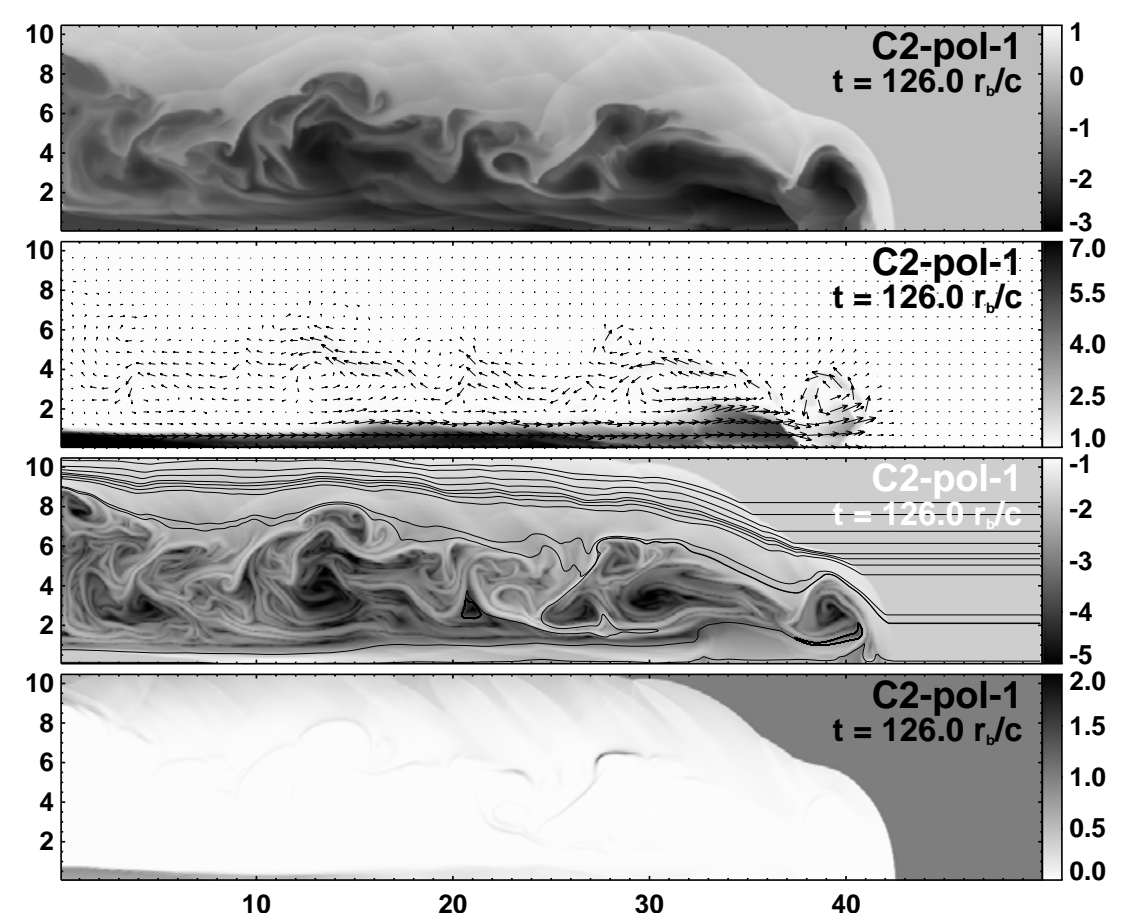

Fig. 5. Snapshots of the poloidal field model C2-pol-1 at $t \sim 126 r_{\mathrm{b}} / c$. From top to bottom: rest mass density, Lorentz factor with flow field, logarithm of the modulus of the magnetic field with field lines superimposed, and magnetization parameter $\beta$. The magnetic field lines shown in the third panel are placed randomly, but weighted with the local field strength, such that on average their density reflects the field strength in the plane where the integration is started $\left(z=50 r_{\mathrm{b}}\right)$.

C2-0. Furthermore, the value of $\beta$ decreases with increasing axial distance along the beam in model C2-pol-1 (Fig. 5) while it is rather uniform in model $\mathrm{C} 2-1$.

The cocoon is also much more homogeneous in model C2-pol-1 than in the other models of series C2, which is especially apparent when comparing the rest mass density snapshots in Figs. 1 and 5. The poloidal field model shows much less turbulent structure, and the back flow of beam material through the cocoon is almost straight (Fig. 5, third panel) indicating that the poloidal magnetic field reduces or completely suppresses the development of Kelvin-Helmholtz instabilities at the surface between the cocoon and the shroud. This is very different in the toroidal field and purely hydrodynamic cases, where the back flow is less ordered.

In model C2-pol-1 the magnetic field is almost completely expelled from the cocoon. The third panel of Fig. 5 shows that the absolute value of the field strength remains very low. The narrow dark filaments in the cavity interior of model C2-pol-1 (Fig. 5, third panel from top) trace the magnetopauses, i.e., the surfaces where the magnetic field changes direction and hence is zero. The magnetopauses are surrounded by areas of high magnetic field strength giving rise to the extended, twisted filaments. The magnetization parameter (Fig. 5, bottom panel) is nearly zero everywhere within the jet, because the magnetic field is advected out the cavity by the fluid flow, and accumulates in the shroud with its large thermal pressure yielding a low value of $\beta$ (compared to e.g., model $\mathrm{C} 2-1$ ). This differs from the behavior of models with toroidal magnetic fields where the magnetic tension prevents a strong reduction of the magnetic field in the cavity.

\subsubsection{C2: Temporal evolution}

The top panel of Fig. 7 displays the motion of the jet's head for all models of series $\mathrm{C} 2$ and a straight line corresponding to the $1 \mathrm{D}$ velocity estimate given by Eq. (47). Apart from model C2-10/3 the jet propagates at essentially the same speed in all models. The $1 \mathrm{D}$ estimate gives a very good approximation to the speed in the 1D phase, and becomes an upper bound in the $2 \mathrm{D}$ phase. The small differences between the analytic estimate for non-magnetized jets and the actual simulation values are due to the fact that the jets are cold. Even the equipartition magnetic energy density (model C2-1) is still very small compared to the ram pressure at the head of the jet. Remarkably, there is no simple dependence of the propagation speed on the magnetic field strength: in model $\mathrm{C} 2-10 / 3$ the jet propagates fastest, in model $\mathrm{C} 2-1$ slowest, in model C2-1/20 second fastest, and the remaining models fall in between. The propagation speed of magnetized jets is determined by a number of competing effects related to both the strength and the topology of the magnetic field. However, our simulations do not show a clear trend with these two parameters, i.e., the speed depends on both parameters nonlinearly. The high speed of model C2-10/3 can be explained by its large nose cone.

The speed of propagation of the Mach disk, $v_{\mathrm{M}}$ (which corresponds, approximately, to the speed of propagation of the jet without considering the nose cone) tends to decrease with increasing magnetization (Table 2 ). In the hydrodynamic model $\mathrm{C} 2-0, v_{\mathrm{M}}$ is slightly smaller than in the weakly magnetized model. This trend also holds for the models of series B1, but not for those of series $\mathrm{C} 1$. 

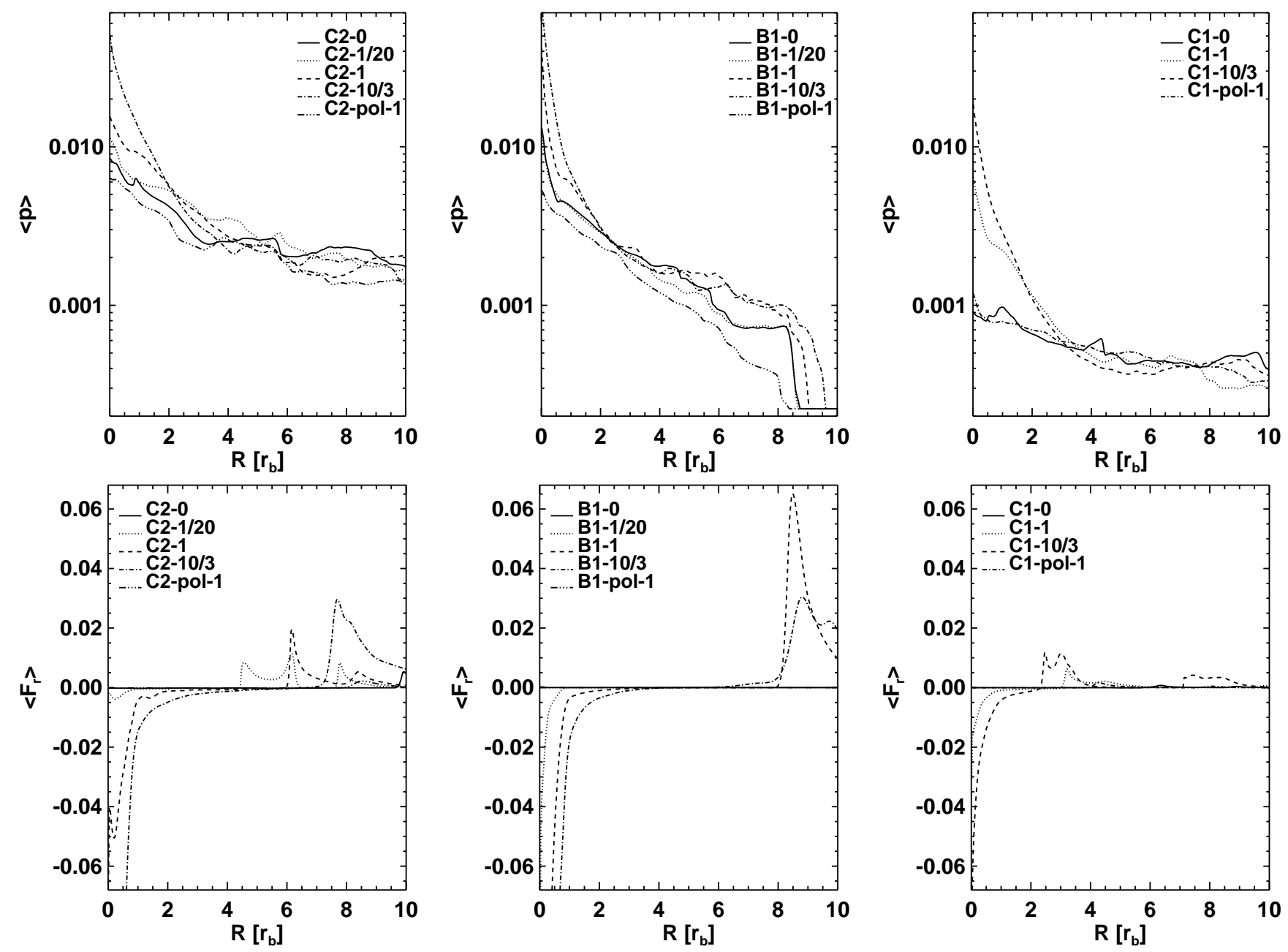

Fig. 6. Average thermal pressure $\langle p\rangle \equiv \sum_{z_{k}=1}^{z_{k}=n_{z}} p\left(r_{i}, z_{k}\right) / n_{z}$ measured in code units (top row) and average of the radial component of the net force $\left\langle F_{r}\right\rangle \equiv \sum_{z_{k}=1}^{z_{k}=n_{z}}\left[-\nabla p\left(r_{i}, z_{k}\right)+(\nabla \times B) \times B\right]_{r} / n_{z}$ (bottom row) versus radius for the models of all series. The snapshots are computed at $t \approx 126 r_{\mathrm{b}} / c$, $\approx 80 r_{\mathrm{b}} / c$ and $\approx 249 r_{\mathrm{b}} / c$ for series C2, B1 and C1 (from left to right), respectively. For models with zero magnetic field and with poloidal fields, the net force is almost zero, and hence the corresponding lines show almost no variation (around zero) at the scale used in the plots in the bottom row.

Table 2. Propagation velocity of the Mach disc $\left(v_{\mathrm{M}}\right)$ for models with a toroidal magnetic field.

\begin{tabular}{lr}
\hline \hline Model & $v_{\mathrm{M}}$ \\
\hline $\mathrm{C} 2-0$ & 0.32 \\
$\mathrm{C} 2-1 / 20$ & 0.33 \\
$\mathrm{C} 2-1$ & 0.29 \\
$\mathrm{C} 2-10 / 3$ & 0.29 \\
\hline $\mathrm{B} 1-0$ & 0.59 \\
$\mathrm{~B} 1-1 / 20$ & 0.61 \\
$\mathrm{~B} 1-1$ & 0.49 \\
$\mathrm{~B} 1-10 / 3$ & 0.42 \\
\hline $\mathrm{C} 1-0$ & 0.10 \\
$\mathrm{C} 1-1$ & 0.10 \\
$\mathrm{C} 1-10 / 3$ & 0.10 \\
\hline
\end{tabular}

Figure 8 shows the cylindrical aspect ratio of all models as a function of time. As the jet reaches the radial grid boundary in models $\mathrm{C} 1$ and $\mathrm{C} 2$ before the end of the simulation, the aspect ratio approaches that of the grid, which is about 5 .
This and the fact that the differences among the models are smaller than those for a computational domain containing the whole cavity, makes one to interpret these plots with caution. Nevertheless, our explanation for the high propagation speed of model C2-10/3 is further supported by its large aspect ratio. The other models do not show any trend.

The average thermal pressure $\langle p\rangle$ (defined as $\langle p\rangle \equiv$ $\left.\sum_{z_{k}=1}^{z_{k}=n_{z}} p\left(r_{i}, z_{k}\right) / n_{z}\right)$ of the more strongly magnetized models (poloidal or toroidal) of series $\mathrm{C} 2$ is up to 4 times larger inside the beam at $t \approx 126 r_{\mathrm{b}} / c$ than in the corresponding hydrodynamic model C2-0 (Fig. 6). The pressure distributions show a distinct maximum at the beam axis instead of being flat there and leveling off at larger radii as predicted by Begelmann et al. (1984). This difference arises because in our simulations the magnetization radius $r_{\mathrm{m}}$ is smaller than the jet radius $\left(r_{\mathrm{m}}=0.37 r_{\mathrm{b}}\right)$, while Begelmann et al. (1984) assume a jet with a uniform current along the whole beam, i.e., $r_{\mathrm{m}}=r_{\mathrm{b}}$. Our simulations further show that the average thermal pressure $\langle p\rangle$ can be dominated by the contributions of the hotspot and/or the nose cone, while Begelmann et al. (1984) exclude these regions 

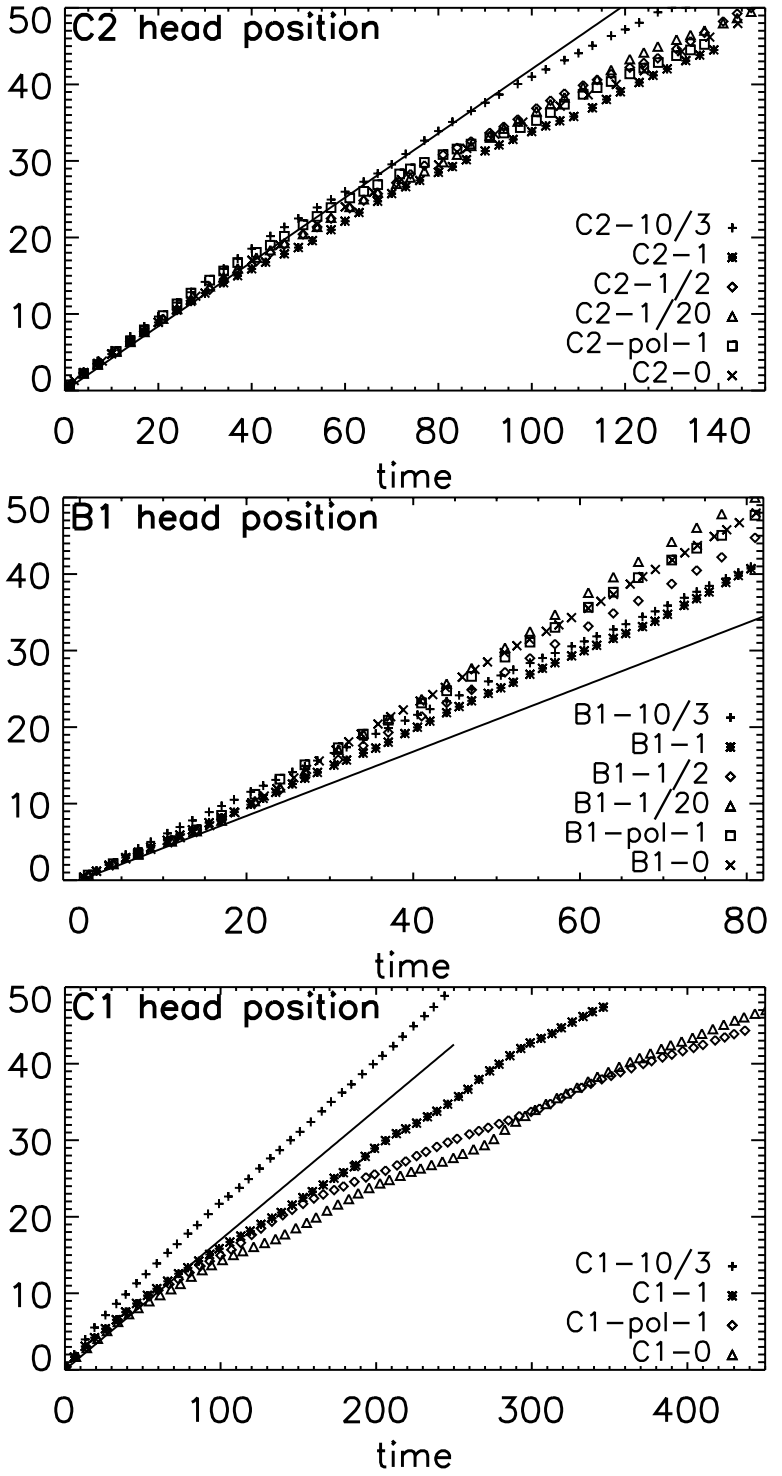

Fig. 7. Motion of the head of the jet for all computed models. The solid lines correspond to the 1D velocity estimate given by Eq. (47).

from their analysis. Just outside the beam $(1 \lesssim r \lesssim 2)$ the radial distribution of $\langle p\rangle$ is quite flat in all models of series $\mathrm{C} 2$ (except of model C2-10/3), and declines at larger radii $(r \gtrsim 2)$. Thus, the jets have a core-sheath structure in radial direction. The radial pressure gradient is larger in model C2-10/3 than in any other model of series $\mathrm{C} 2$ and seems to decrease monotonically with decreasing magnetization. Beyond some typical radius $\left(\approx 4 r_{\mathrm{b}}\right)\langle p\rangle$ as a function of $r$ becomes very similar (almost flat) for all the models. Also the thermal pressure gradients $(\nabla p)$ approach to zero beyond $\approx 4 r_{\mathrm{b}}$. The behavior of model C2-pol-1 is different from that of the models transporting toroidal fields. Its average thermal pressure is smaller than that in the hydrodynamic model $\mathrm{C} 2-0$ at all radii.

The high compression of the beam in models carrying a toroidal magnetic field is the result of the imposed magnetic field structure in the beam (Eq. (44)). Up to the magnetization radius $r_{\mathrm{m}}, b^{\phi}$ grows linearly with radius, i.e., the pressure
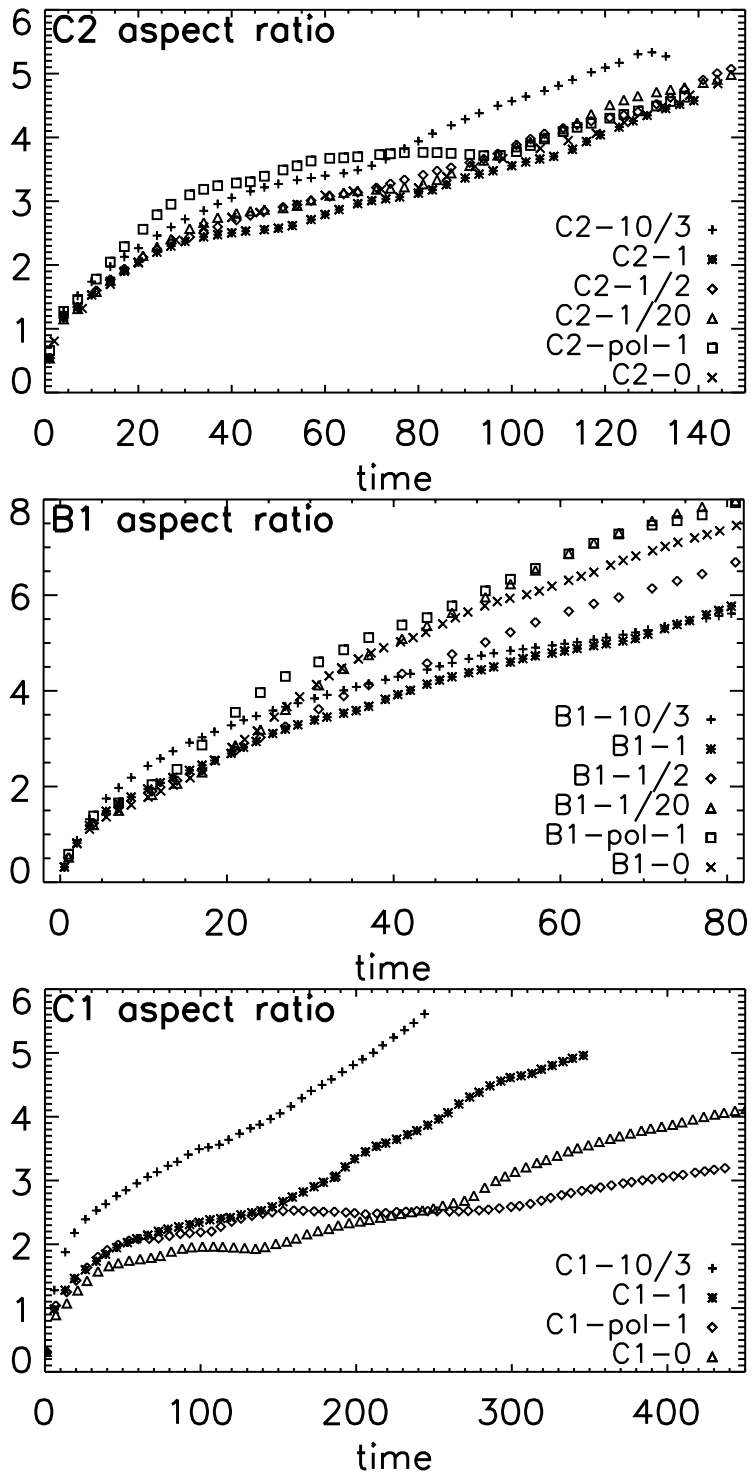

Fig. 8. Temporal change of the cylindric aspect ratio $A_{\mathrm{c}}$ for all models.

necessary to keep hydromagnetic equilibrium for $r<r_{\mathrm{m}}$ is (e.g. Lind et al. 1989)

$p(r)=\left[\alpha+2 \beta_{\mathrm{m}}\left(1-\frac{r^{2}}{r_{\mathrm{m}}^{2}}\right)\right] p_{\mathrm{e}}$,

where $\alpha$ is a constant, and $\beta_{\mathrm{m}} \equiv\left(b_{\mathrm{m}} W_{\mathrm{m}}\right)^{2} /\left(8 \pi p_{\mathrm{e}}\right)=(1-$ $\alpha)\left(r_{\mathrm{b}} / r_{\mathrm{m}}\right)^{2}$. Hence, the pressure distribution has its maximum at $r=0$.

Models $\mathrm{C} 2-1 / 20, \mathrm{C} 2-1$ and $\mathrm{C} 2-10 / 3$ which transport toroidal magnetic fields are confined by the toroidal fields in the beam and in the cocoon. In the cocoon the density profile rearranges itself in such a way that the toroidal field decreases nearly linearly with radius (note that this scaling does not hold inside the magnetization radius $r_{\mathrm{m}}$ ). The radial distribution of the axial average of the net radial force $\left\langle F_{r}\right\rangle \equiv$ $\sum_{z_{k}=1}^{z_{k}=n_{z}}\left[-\nabla p\left(r_{i}, z_{k}\right)+(\nabla \times B) \times B\right]_{r} / n_{z}$ (Fig. 6) displays two distinct regions in case of models with toroidal fields (for model C2-pol-1 $\left\langle F_{r}\right\rangle \approx 0$ ): (1) an internal (contractive region up to $\approx 4 r_{\mathrm{b}}$ ) where $\left\langle F_{r}\right\rangle<0$ and where the gas in the beam and its 


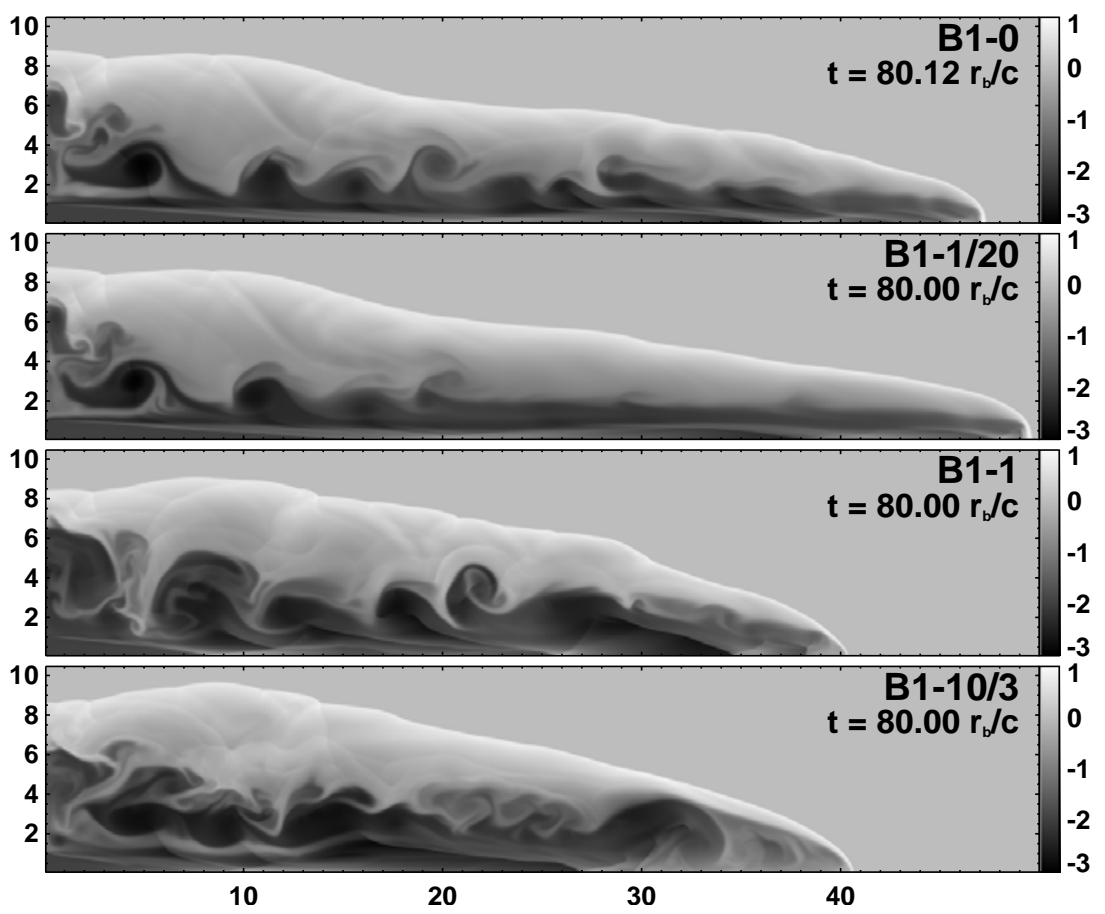

Fig. 9. Snapshots of the logarithm of the rest mass density of models B1-0, B1-1/20, B1-1 and B1-10/3 at $t \sim 80 r_{\mathrm{b}} / c$. For model B1-1/20 the jet is about to leave the computational grid because of its large propagation speed.

neighborhood is pinched; and (2) an external (expansive region), where $\left\langle F_{r}\right\rangle>0$ because the thermal pressure gradient dominates the magnetic tension force.

\subsection{Series $B 1$}

\subsubsection{B1-0 to B1-10/3: Purely hydrodynamic and toroidal field models}

The results of the non-magnetized and toroidal field models of the series B1 are displayed in Figs. 9 and 10. The rest mass density (Fig. 9) shows some of the trends of series $\mathrm{C} 2$, however the effects caused by the toroidal magnetic field do not scale linearly with the magnetic field strength. The weakly magnetized model B1-1/20 behaves quite differently both from the equipartition model B1-1 and the strongly magnetized model B1-10/3. The more magnetized models develop nose cones preceded in the case of model B $1-10 / 3$ by a magnetically confined bubble. The rest mass density (Fig. 9) and the pressure (not shown) distributions show much more structure for these two more strongly magnetized models than that of the nonmagnetized model. The corresponding distributions of model B1-1/20 are very smooth suggesting that its weak magnetic field inhibits the formation of structures (see Sect. 5).

The first recollimation shock shows the same trend as in series $\mathrm{C} 2$. Due to magnetic pinching the shock is stronger and is located closer to the nozzle for a stronger toroidal field. The strong pinching of the beam in models B $1-1$ and B1-10/3 leads to a widening of the beam downstream of the recollimation shocks and to smaller Lorentz factors ( $W \sim 5$; Fig. 10). In model $\mathrm{B} 1-1 / 20$ the pinching is very weak, and no difference to the non-magnetized model can be recognized. Instead, it is even better collimated than model B $1-0$, and the flow remains highly relativistic up to the terminal shock of the beam, where the beam plasma is deflected and flows backwards smoothly in a very thin layer around the beam. This behavior explains why the jet of model B1-1/20 has propagated further than those of the other models in the same time. These results imply, that with the right combination of parameters, a small toroidal field helps to collimate the beam without producing too much pinching, which helps to keep the jet stable.

Figure 11 shows the magnetization $\beta$ and $B^{\phi}$ for the two models $\mathrm{B} 1-1$ and $\mathrm{B} 1-10 / 3$, respectively. As for the corresponding $\mathrm{C} 2$ models the initial magnetization of the beam is reduced very much in the first cross shock. It is strongest near the beam's terminal shock, where the plasma flows radially away from the axis leading to the formation of the nose cone. Apart from the fact that the structures are much narrower than in the models of series $\mathrm{C} 2$, the overall distributions of $\beta$ and $B^{\phi}$ are very similar everywhere in the cavity. $\beta$ is lowest near the axis and then increases until it reaches its maximum value in the magnetic shell around the beam. $B^{\phi}$ remains largest inside the beam and the nose cone. Different from series $\mathrm{C} 2$, an increase of the magnetic field strength yields a more turbulent cocoon in case of the models of series B1.

\subsubsection{B1-pol-1: Poloidal field model}

Figure 12 shows snapshots of the rest mass density, the Lorentz factor, the magnetic field strength and the magnetization parameter of model B1-pol-1. Most of the features found in model C2-pol-1 are also present here: the jet as a whole is very smooth both in density and pressure, the cocoon showing only an indication of an eddy. The beam is almost undisturbed, 


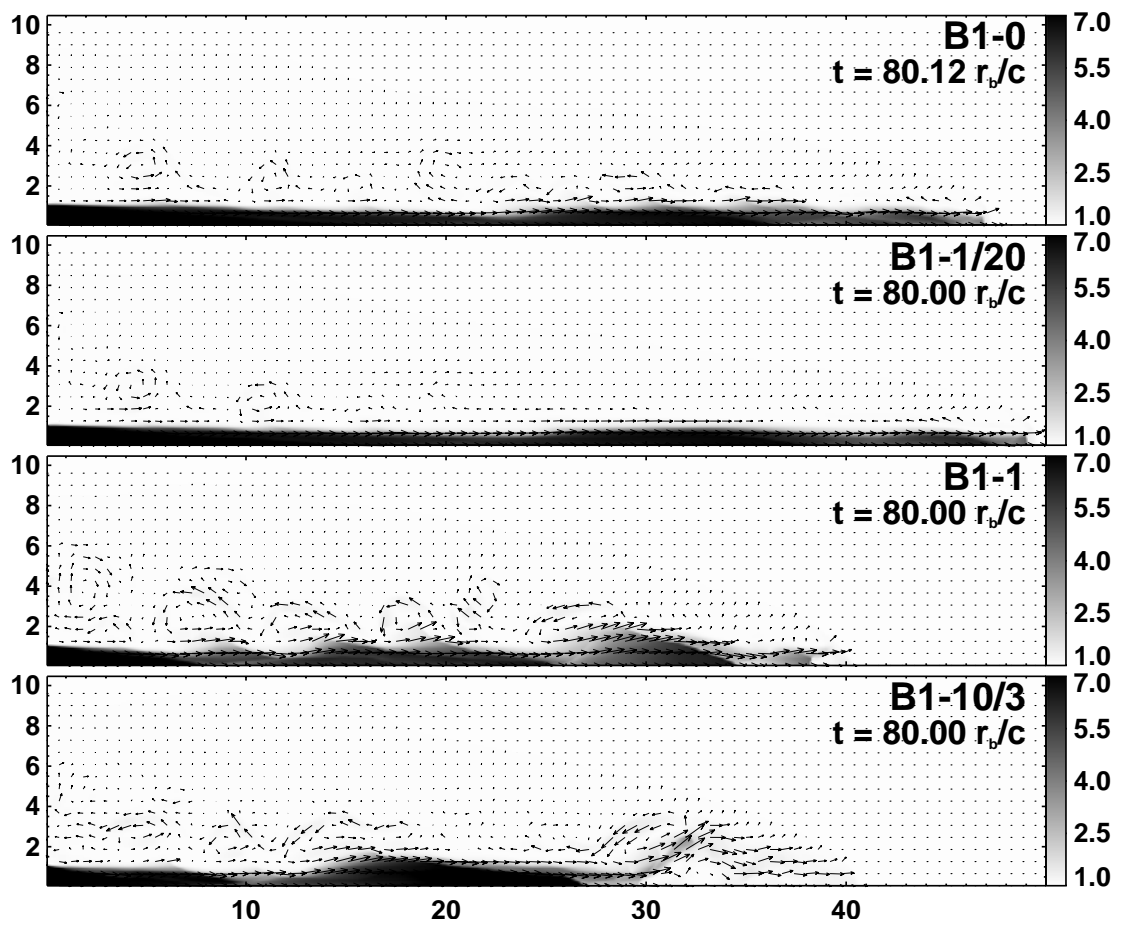

Fig. 10. Same as Fig. 9 but showing snapshots of the Lorentz factor and the flow field.

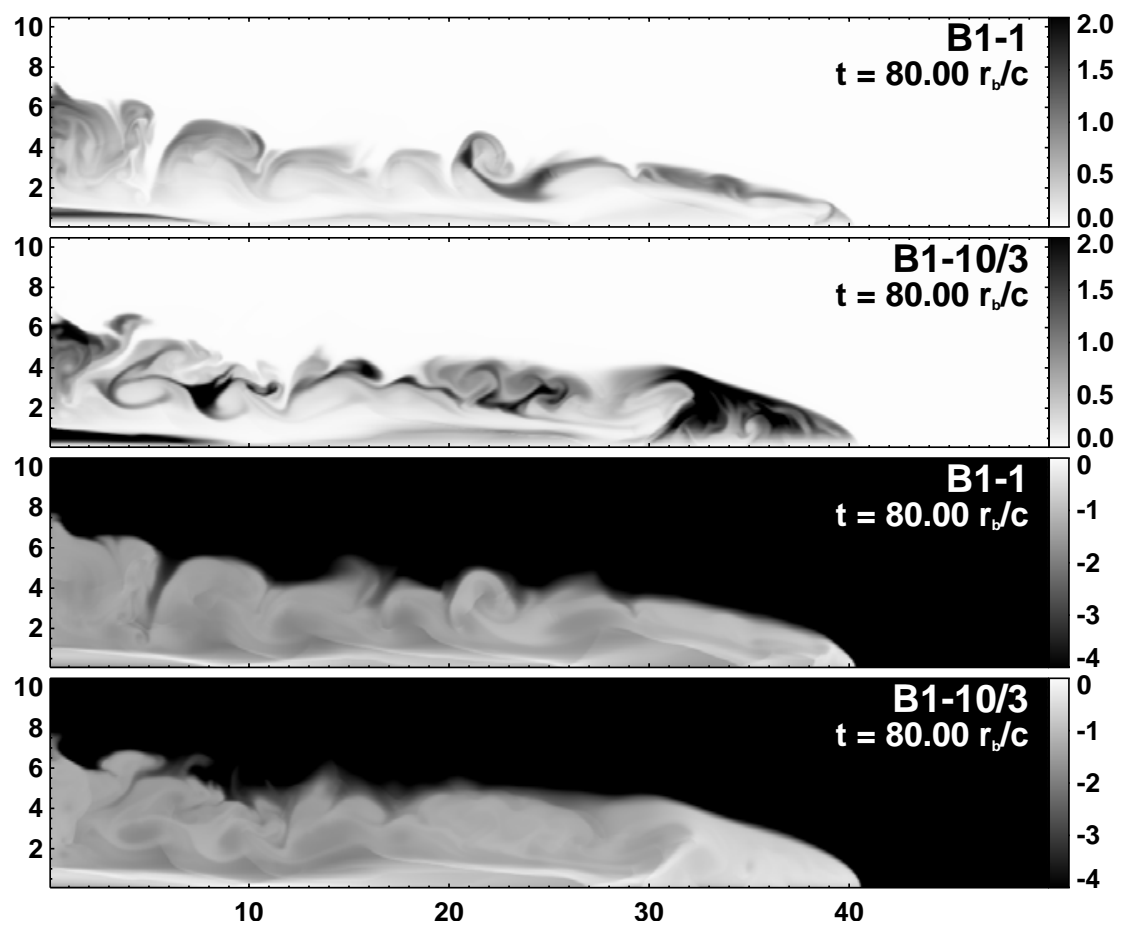

Fig. 11. Snapshots of the magnetization parameter $\beta$ (upper two panels) and the toroidal field component $B^{\phi}$ (lower two panels) of the two most strongly magnetized models of series B1 at $t \sim 80 r_{\mathrm{b}} / c$.

and the cross shocks are very weak. The back flow is confined to a narrow region directly around the beam and is absolutely straight. The hammer-like structure at the head of the jet seen in model C2-pol-1, which is associated with the emission of vortices, is not present in model B1-pol-1.

The smoothness of the cocoon results from the presence of the poloidal field (see the top panel of Fig. 12), which is smooth throughout the cavity. The field is only twisted in the narrow low density sheet around the beam, and even there, the twist is mostly aligned with the axis. This does not favor the formation of eddies, which could drive strong shocks into the beam. As in model $\mathrm{C} 2-$ pol- $1, \beta \approx 10^{-6}$ in a large part of the cavity, which however, does not diminish the effect of the poloidal field on the morphology of the jet. Model B1-pol-1 shows a smoother 


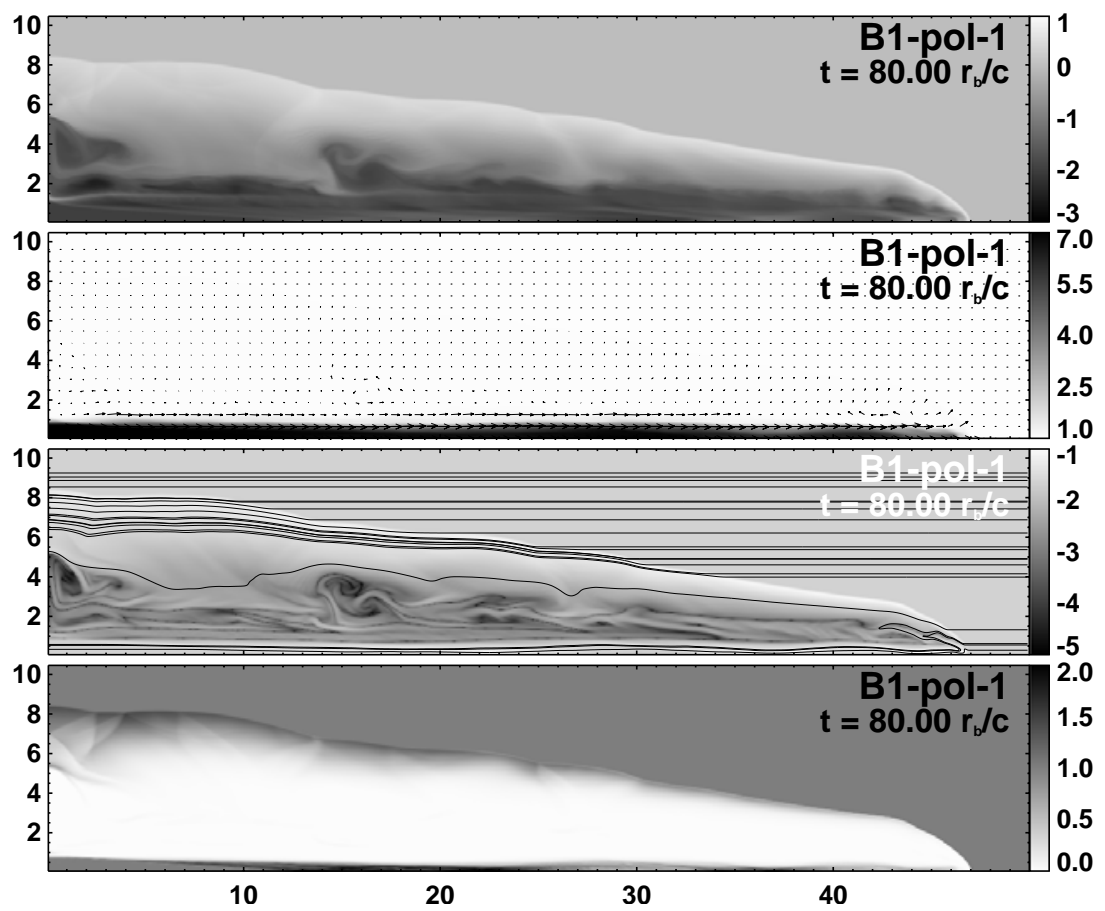

Fig. 12. Snapshots of the poloidal field model B1-pol-1 at $t \sim 80 r_{\mathrm{b}} / c$. From top to bottom: rest mass density, Lorentz factor with flow field, logarithm of the modulus of the magnetic field with field lines superimposed, and $\beta$. The magnetic field lines shown in the third panel are placed randomly, but weighted with the local field strength such that on average their density reflects the field strength in the plane where the integration is started $\left(z=50 r_{\mathrm{b}}\right)$.

cavity than model $\mathrm{C} 2-$ pol-1 because the cocoon (even in the hydrodynamic case) is less prominent in series B1 than in series $\mathrm{C} 2$ (see Sect. 4.2.3).

\subsubsection{B1: Temporal evolution}

Models C2-0 and B1-0 differ only by the adiabatic index (see Table 1, yet the cavity of the latter is much more elongated and the jet has a larger propagation speed. These differences can be understood using the simple analytic model of Begelman \& Cioffi (1989). In their model, the cavity pressure $P_{\mathrm{c}}$ determines the sideways expansion of the cocoon. This pressure is related to $\gamma$, the propagation velocity of the bow shock in the axial direction $\left(v_{\mathrm{h}}\right)$, the cavity cross section $\left(F_{\mathrm{c}}\right)$, and the power transported into the cocoon through the Mach disc $\left(L_{\mathrm{c}}\right)$ by

$P_{\mathrm{c}}=\frac{(\gamma-1) L_{\mathrm{c}}}{v_{\mathrm{h}} F_{\mathrm{c}}}$.

In series $\mathrm{C} 2$ the quantity $\gamma-1$ is twice as large as in series B1. Therefore, the sideways expansion velocity of the cocoon $\left(v_{\mathrm{c}} \propto(\gamma-1)^{1 / 4}\right)$ of model B1 is about $20 \%$ smaller than in model C2. However, this alone does not explain the expansion velocity differences found in the simulations. One also has to consider that the smaller expansion velocities of the cocoons of series B1 yield bow shocks whose angles with respect to the direction of propagation of the jet are smaller. As an oblique shock is less efficient than a normal shock in decelerating the jet, the propagation velocity of the jet is faster than expected from the one-dimensional estimate, Eq. (47). Thus, the effective energy flux through the Mach disk is smaller as the flux is proportional to the speed difference between the beam and the head, $L_{\mathrm{c}} \propto\left(v_{\mathrm{b}}-v_{\mathrm{h}}\right)$. Consequently, the pressure in the cocoon grows less, i.e., it also expands less in the transverse direction. The two processes feedback each other, and explain the narrow and pointy cavities blown by the models of series B1.

We now focus on the effects of the magnetic field on the propagation of the jets in series B1. As discussed in the previous paragraph, the 1D estimate (Eq. (47)) is a lower bound of the propagation velocity, and as for series $\mathrm{C} 2$, the propagation speed does not depend linearly on the magnetic field strength (Fig. 7). However, in contrast to the $\mathrm{C} 2$ jets, the jet with the weakest toroidal field propagates fastest followed by the hydro model and the poloidal field model. Consequently, due to their larger propagation velocities, these models also exhibit larger aspect ratios (Fig. 8). The poloidal field model B1-pol1 is more elongated than model $\mathrm{B} 1-1 / 20$, because the poloidal field initially present in the ambient medium inhibits the sideways expansion of the cavity. The jet of model B1-pol-1 propagates $\approx 20 \%$ faster than that of model B1-1, which has a similar degree of magnetization, because of the reduced transversal expansion of the cocoon (according to the argument expressed above).

The average thermal pressure of the models of series B behaves qualitatively similarly, but is slightly larger than that of the corresponding models of series C2 (Fig. 6). Furthermore, the distance at which all the models display a very similar thermal pressure and pressure gradient $\left(\approx 3 r_{\mathrm{b}}\right)$ is slightly smaller than in the $\mathrm{C} 2$ series (with the exception of B1-pol-1). The models of series B1 with a toroidal field develop a region in the cocoon between the contractive and the expansive regions (see above), where there is an almost perfect 


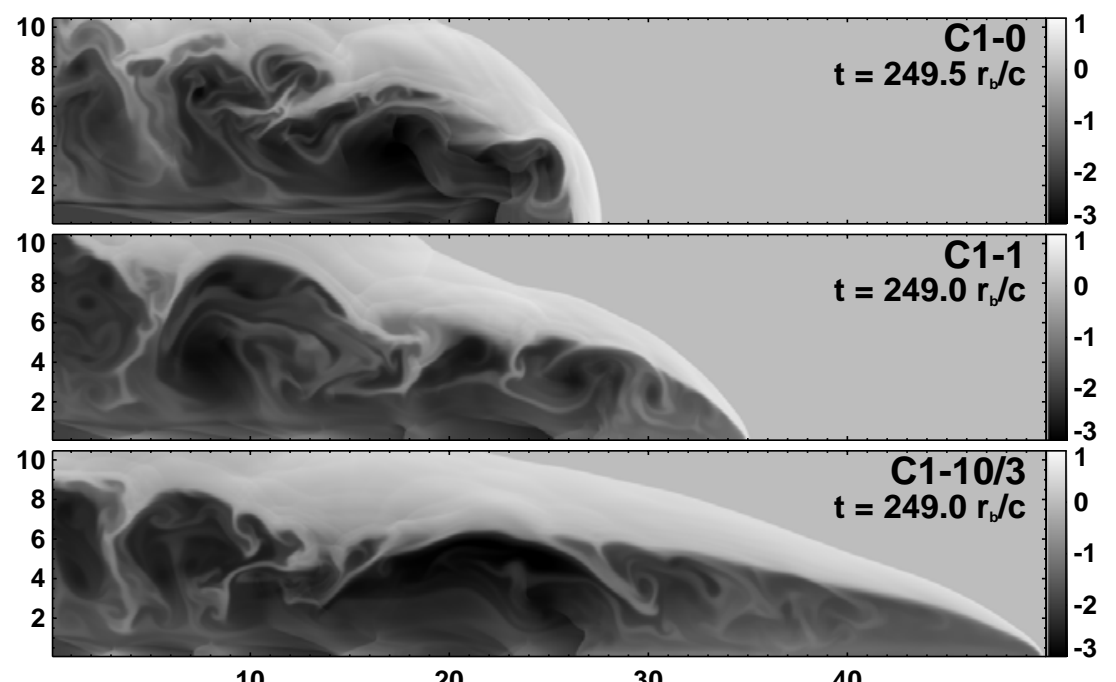

10

20

30

40

Fig. 13. Snapshots of the logarithm of the rest mass density of the models $\mathrm{C} 1-0, \mathrm{C} 1-1$ and $\mathrm{C} 1-10 / 3$ at $t \sim 249 r_{\mathrm{b}} / c$.

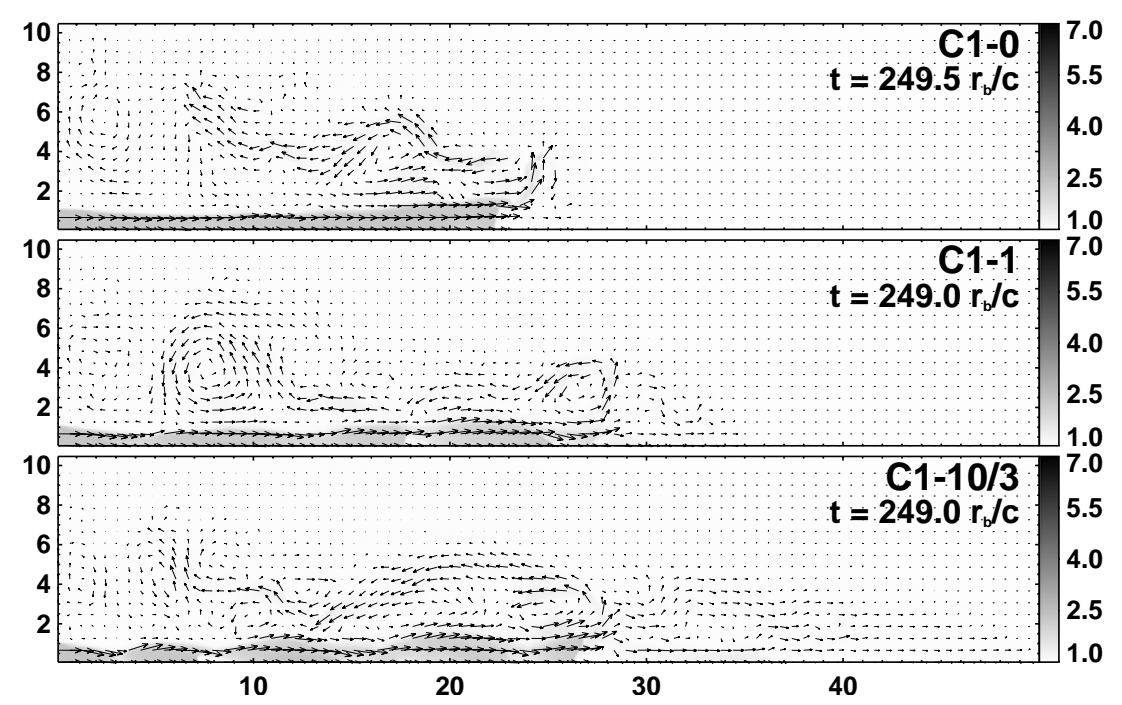

Fig. 14. Same as Fig. 13 but showing snapshots of the Lorentz factor and the flow pattern.

hydromagnetic equilibrium the net force being almost zero (Fig. 6). Model B1-pol-1 differs in its behavior, because $\langle p\rangle$ is everywhere smaller than in the hydrodynamic model B1-0 and decreases almost exactly as $r^{-1}$.

\subsection{Series C1}

\subsubsection{Toroidal and poloidal field models}

Figures 13 and 14 show the rest mass density and the Lorentz factor, respectively, of all the models of series $\mathrm{C} 1$ with toroidal magnetic fields. Many of the effects of the different magnetic field configurations described in the previous sections also hold for series $\mathrm{C} 1$, some of them being more enhanced.

The toroidal field models develop very large nose cones. In model $\mathrm{C} 1-10 / 3$ the nose cone makes up $50 \%$ of the total length of the jet, which has a high density $\left(\rho \sim 6 \rho_{\mathrm{b}}\right)$, high pressure $\left(p \sim 200 p_{\mathrm{b}}\right)$ spine consisting of beam plasma preceded by a large low density bubble filled up with beam material.
The same structure is visible in model $\mathrm{C} 1-1$, although the nose cone is only half as long as in model C1-10/3 (Fig. 13).

As in series B1 and $\mathrm{C} 2$, the strength of the recollimation shocks increases with toroidal magnetic field, because most of the cocoon of models $\mathrm{C} 1-1$ and $\mathrm{C} 1-10 / 3$ is dominated by magnetic pressure (see upper two panels of Fig. 15). This also explains the high density of the nose cone of model C1-10/3 (Fig. 13), which is confined by strongly magnetized matter. The strong recollimation shocks reduce the Lorentz factor in the post-shock regions to values close to 1 . In the most strongly magnetized model of the series $(\mathrm{C} 1-10 / 3)$ some of the recollimation shocks are planar instead of conical shocks (Fig. 4, bottom panel), i.e., the beam fluid is most effectively decelerated (Fig. 14). In the magnetized models the back flow of beam plasma through the cocoon is confined by the toroidal field, i.e., it is slower and smoother than in model C1-0 (Fig. 14).

Model C1-pol-1 also shows the same, but enhanced features as model C2-pol-1. The density and pressure morphology look very similar to that of model $\mathrm{C} 1-0$, which has a 


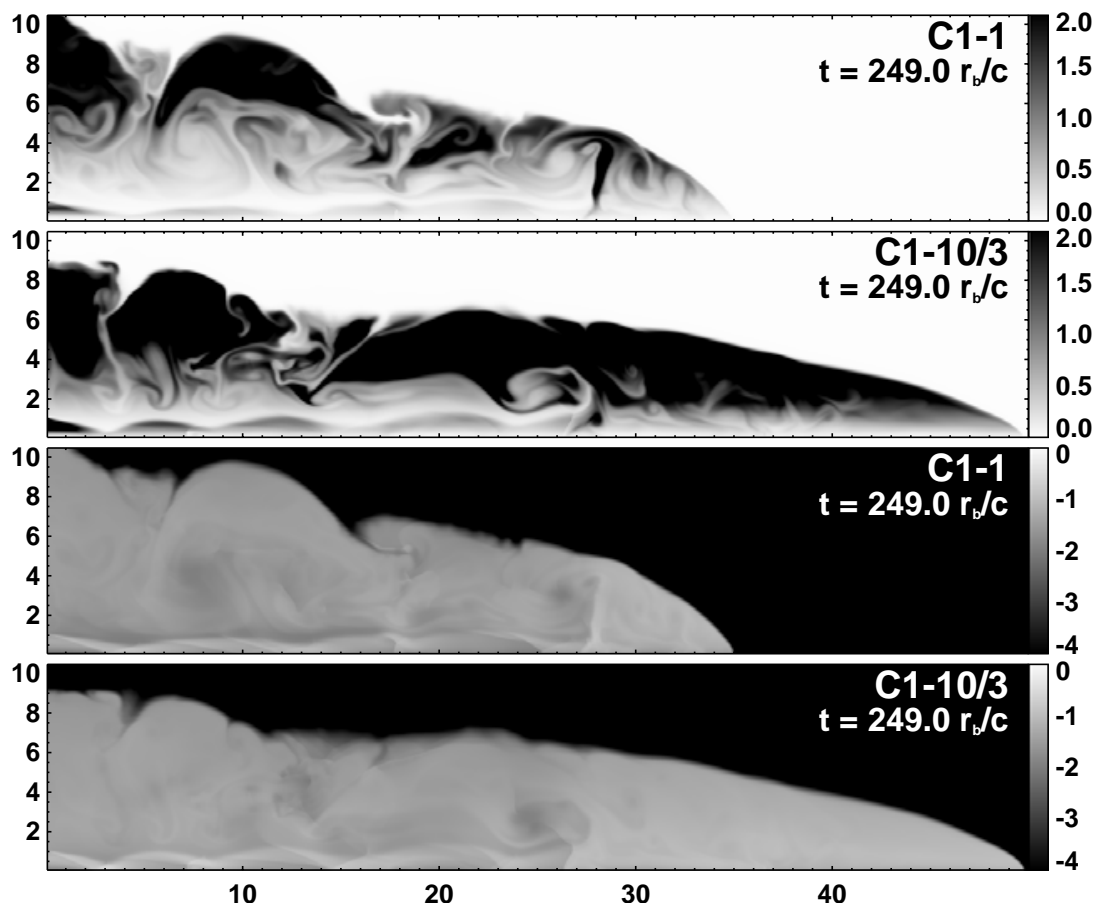

Fig. 15. Snapshots of the magnetization parameter $\beta$ (upper two panels) and the toroidal field component $B^{\phi}$ (lower two panels) of the two most strongly magnetized models of series C1 at $t \sim 249 r_{\mathrm{b}} / c$.

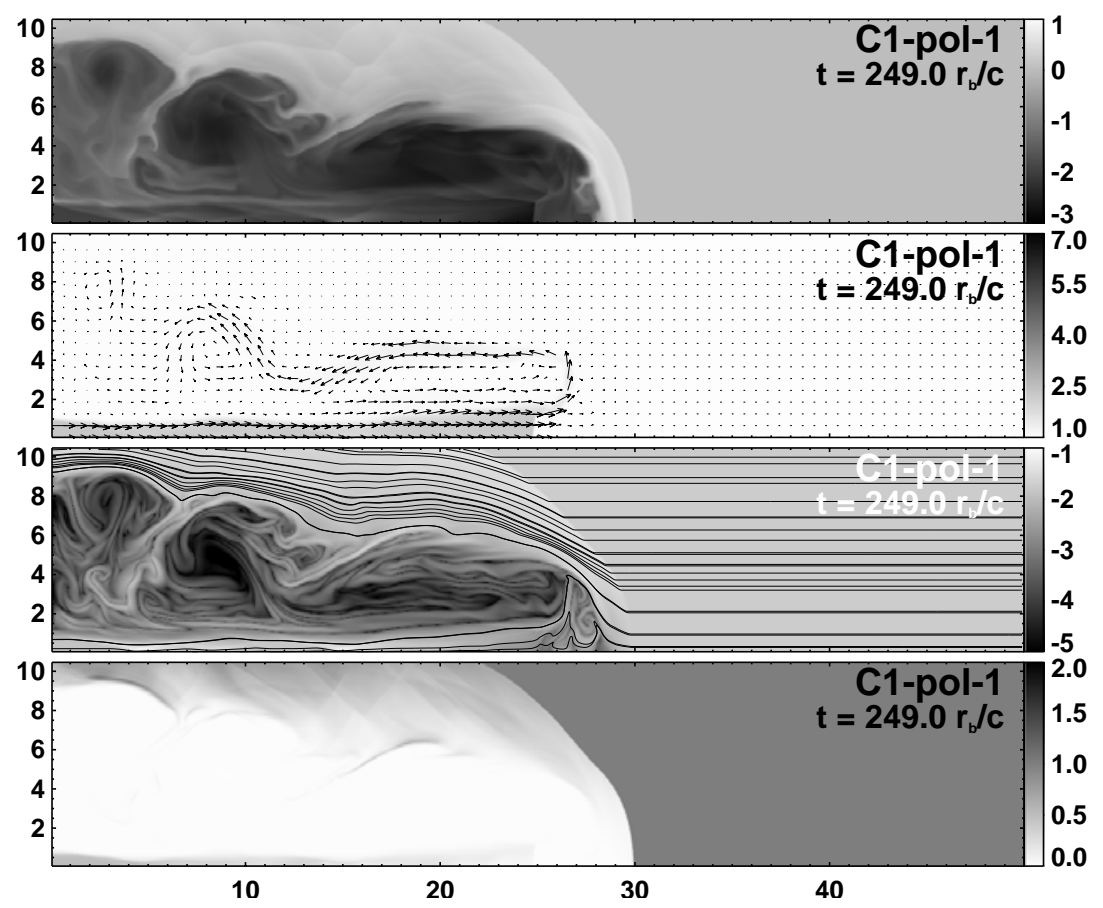

Fig. 16. Snapshots of the poloidal field model C1-pol-1 at $t \sim 249 r_{\mathrm{b}} / c$. From top to bottom: rest mass density, Lorentz factor with flow field, logarithm of the modulus of the magnetic field with field lines superimposed, and magnetization parameter $\beta$. The magnetic field lines shown in the third panel are placed randomly, but weighted with the local field strength, such that on average their density reflects the field strength in the plane where the integration is started $\left(z=50 r_{\mathrm{b}}\right)$.

similar propagation speed, too. However, the cross shocks are weaker than in the non-magnetized model, and the pressure in the cocoon is slightly more homogeneous. The flow pattern (second panel from top in Fig. 16) shows the same hammer-like head structure as observed in model C2-pol-1
(Sect. 4.1.2). Model $\mathrm{C} 1-$ pol-1 has the same magnetization structure as model C2-pol-1, but the shell surrounding its beam is more strongly magnetized (bottom panel of Fig. 16). The magnetic field is almost completely expelled from the jet's cocoon, and is reduced by four to five orders of magnitude 
compared to the initial value (third panel from top in Fig. 16). Only twisted filaments of larger magnetic field strength survive inside the cocoon.

\subsubsection{C1: Temporal evolution}

All models propagate at a speed very close to the $1 \mathrm{D}$ estimate (Eq. (47)) up to an evolutionary time of $t \approx 80 r_{\mathrm{b}} / c$ (1D phase; see Fig. 7, top panel). Only model C $1-10 / 3$ propagates faster from the very beginning of its evolution, and shows no sign of slowing down during the whole simulation. The other three models eventually leave the $1 \mathrm{D}$ phase and start slowing down. Model C1-1 is still faster than model C1-pol-1 and the nonmagnetized models, the latter two following a similar evolutionary track. We find that the toroidal magnetic field speeds up the jet by almost $100 \%$ in the case of model C1-10/3 and by about $20 \%$ in the case of model $\mathrm{C} 1-1$ as compared to model C1-0. However, this effect is not seen for the velocities of the Mach disk (Table 2). The poloidal field does not have a large effect on the propagation speed of the jet. Model C1-pol-1 shows only a slightly stronger deceleration compared to model $\mathrm{C} 1-0$ at the very end of the simulation.

The models of series $\mathrm{C} 1$ with a toroidal field develop larger nose cones than the equivalent models of series $\mathrm{C} 2$, because of the smaller propagation velocity of the former. For smaller propagation speeds there is a larger amount of mass crossing the Mach disk which ends up in the cavity, and hence there is also a larger amount of matter that is deflected forward and fills up a larger nose cone. In other words, the nose cone is larger in those models where the cocoon is larger (i.e., in models where the speed of the head is smaller).

As with series $\mathrm{C} 2$ the cylindrical aspect ratio (bottom panel in Fig. 8) has to be analyzed with caution, since in all models the cavity reaches the edge of the grid in radial direction quite early in the simulation. Nevertheless, the differences between the models are larger than suggested by their propagation speeds alone. While model $\mathrm{C} 1-10 / 3$ has a much larger aspect ratio than any of the other models, because of its long nose cone, the two equipartition models, C1-1 and C1-pol-1, have similar aspect ratios for $t \lesssim 140 r_{\mathrm{b}} / c$. Later model C1-1 evolves differently, and its aspect ratio almost doubles until the end of the simulation. Model C1-pol-1, however, maintains a constant aspect ratio of around 2.4 until it hits the radial boundary of the grid at $t \approx 300 r_{\mathrm{b}} / c$. The aspect ratio of model $\mathrm{C} 1-0$ is even smaller in the beginning, but starts to grow earlier in the simulation.

Although the thermal pressure of the gas injected through the nozzle is quite similar in all three series, the averaged thermal pressure along the beam of the models of series $\mathrm{C} 1$ is smaller than that of series $\mathrm{C} 2$ or $\mathrm{B} 1$, if one compares models with the same $\bar{\beta}$ (Fig. 6). The reason is that the averaged pressure along the axis is dominated by the contribution of the hotspot or the plug pressure, which is much smaller in series $\mathrm{C} 1$ than in series $\mathrm{C} 2$ or B1. However, the relative increase of the averaged gas pressure of models $\mathrm{C} 1-1$ and $\mathrm{C} 1-10 / 3$ relative to the hydrodynamic model $\mathrm{C} 1-0$ is roughly twice times larger than in the other two series.

\section{Discussion and conclusions}

Our results show that jets carrying a toroidal field are confined both by the field transported inside the beam and by the field advected into the cocoon by the back flow. The most prominent morphological feature caused by the toroidal magnetic field is the nose cone. As found in previous Newtonian (e.g. Clarke et al. 1986; Lind et al. 1989; Kössl et al. 1990) and relativistic simulations (Komissarov 1999b) this structure occurs for jets with toroidal fields close to equipartition strength or larger and for $\sigma \gtrsim 0.01$. The nose cone forms because of the magnetic pinching by the Lorentz force which acts inwards radially near the beam. The magnetized beam plasma (which in a non-magnetized beam is deflected at the Mach disk shock and flows backwards inflating the cocoon) is partially forced to flow around the Mach disk into the nose cone. Only a smaller fraction of the beam plasma (namely the less magnetized fraction for which the Lorentz force is not as strong) flows backwards into bubble-like structures that reside upstream of the nose cone forming a cocoon similar as in hydrodynamic jets. This formation mechanism explains several properties of nose cones: (a) the larger the magnetization of the beam plasma, the stronger is the Lorentz force acting on the flow in the beam, i.e., more of the shocked beam plasma is forced into the nose cone which thereby grows larger with larger magnetization of the beam; (b) nose cones contain stronger magnetic fields than cocoons, because only the weakly magnetized plasma flows into the cocoon. The high magnetic field in the nose cone itself is the cause for the confinement to a narrow region, the high pressure (approximately two orders of magnitude higher than the external pressure in the $\bar{\beta}=10 / 3$ models) and high density $\left(\sim 10 \rho_{\mathrm{b}}\right)$ spine.

According to Komissarov (1999b) prominent nose cones should not form in low Poynting flux jets even if the magnetization parameter is large. Our results confirm this argument if we restrict ourselves to models transporting toroidal magnetic fields: nose cones only form in those models with toroidal magnetic field where $\sigma>0.01$ and $\beta \geq 1$ at injection. However, even if these requirements are fulfilled, none of our poloidal field models develops a nose cone. On the other hand, the formation of the nose cone in case of strong toroidal fields resembles what happens in pulsar wind nebulae: the postshock velocity cannot decrease fast enough to match the outer boundary condition. Hence, the region expands mostly longitudinally (see, e.g., Bucciantini et al. 2004). The size of the nose cone is correlated with the propagation velocity of the Mach disc, but neither with $\sigma$ nor $\bar{\beta}$. The most prominent nose cone exists in model $\mathrm{C} 1-10 / 3$ where the propagation velocity of the Mach disk is the smallest of all models, and where $\sigma$ is considerably smaller than in models $\mathrm{B} 1-10 / 3$ or $\mathrm{C} 2-10 / 3$ which have the same $\bar{\beta}$.

Since a considerable fraction of shocked beam plasma is locked in the nose cone, it cannot flow back towards the nozzle and drive the radial expansion of the cavity. However, most of the toroidal field models are not narrower (i.e., have a smaller aspect ratio) than their corresponding hydrodynamic reference 
models. Considering the models of series $\mathrm{B} 1,{ }^{2}$ the aspect ratio is smaller both in models $\mathrm{B} 1-1$ and $\mathrm{B} 1-10 / 3$ than in model B1-0, which is possible because of the subtle relation between the mass flux, the mass accumulated in the nose cone, and the force balance in the radial direction. Let us assume that the pressure and the Lorentz force scale differently with $r$. If $p \propto$ $r^{-m}$ and $B_{\phi} \propto r^{-n}$, the net force will vary with radius as

$$
F_{r}=-\frac{\partial p}{\partial r}-\frac{1}{r^{2}} \frac{\partial\left(r^{2} B_{\phi}^{2}\right)}{\partial r}=k_{1} r^{-m-1}+k_{2} r^{-2 n-1},
$$

where $k_{1}$ and $k_{2}$ are constants. Thus, if $m<2 n$ the thermal pressure gradient will eventually dominate the radial net force at some characteristic radial distance, even if at small radii the magnetic tension force is larger than the thermal pressure force. This is the case in all our toroidal field models. A larger value of the injected toroidal field strength yields a larger magnetic tension in the beam and its immediate neighborhood, which is compensated (and eventually exceeded) by the gradient of the total pressure (the isotropic magnetic pressure also counteracts the magnetic tension). This mechanism explains the paradox that a stronger toroidal field does not result in much narrower jets, even when considering that with a stronger magnetic field the amount of matter in the plug grows, and thus the amount of matter in the cocoon decreases.

In all models transporting toroidal field the aspect ratio of the cavity is very similar to that of the hydrodynamic model B1-0, because the pressure gradient causes an expansion for $r \gtrsim 4 r_{\mathrm{b}}$ which compensates for the smaller mass flux into the cocoon. As a rule of thumb, the net force that contracts the beam and its immediate neighborhood (contractive region) causes an expansion in the outer layers of the cocoon and the cavity (expansive region). The increased gas pressure, the formation of strong shocks in the beam, and the pull of the expanding region limit the amount of the contraction. However, as the balance between the pinch induced by the magnetic tension and the total pressure is not perfect, a number of transient phenomena arise. For example, magnetic bubbles form close to the head of the jet around the Mach disk in models with stronger toroidal fields, because the flow tries to establish hydromagnetic equilibrium in the cocoon. In regions which are evacuated (e.g., because of a strong back flow) the gas pressure decrease tends to be balanced by the growth of the magnetic field, i.e., the reduced thermal pressure is compensated for by an increase of the isotropic magnetic pressure. This mechanism is similar to that described by Parker (1979) when considering an isolated flux tube, but applied to extended regions of the jet cavity. In actual radio sources, the magnetically confined bubbles could be observed as spherical smooth radio lobes surrounding the hot spot. One example of such a radio structure may be the hot spot residing in the middle of the roughly spherical radio lobe associated to the jet in 3C 353 (Swain et al. 1998). Interestingly, the counter jet of the same source shows a striking bright annular feature which could be associated with the flow around

\footnotetext{
2 The cocoons of this model series are fully contained in the computational grid and, thus, the aspect ratios are significant, although the qualitative argument is valid for all the series of models.
}

the beam terminal shock in a jet with a dynamically important toroidal magnetic field.

Opposite trends are observed for the evolution of the aspect ratios of models $\mathrm{C} 1-10 / 3$ and $\mathrm{C} 2-10 / 3$ on the one hand, and model B1-10/3 on the other hand. While all three models have the same strong magnetic field, the nose cone of models $\mathrm{C} 1-10 / 3$ and $\mathrm{C} 2-10 / 3$ are much larger than that of model B1-10/3 leaving a much smaller fraction of high pressure plasma to drive the radial expansion of the cavity.

Owing to the same inward directed Lorentz force which produces the nose cones, the internal cross shocks are stronger in the toroidal field models than in the corresponding hydrodynamic models. In the poloidal field case, however, the effect is opposite: the magnetic field makes the jet less susceptible to shock waves driven into the beam perpendicular to the field lines as the magnetic tension on the beam repels shock waves. While the cross shocks in hydrodynamic jets are produced mainly by compression waves driven into the beam from the outside (e.g., by eddies in the cavity), the cross shocks in the toroidal field models are of different nature. They are created intrinsically by the inwards pointing magnetic stress even in models having smooth cocoons, like e.g., model B1-1/20.

The strengthening of the cross shocks with increasing toroidal magnetic field may cause a more knotty appearance of kpc-scale magnetic jets compared to hydrodynamic ones. The extra pinching provided by the toroidal field leads to a decrease of the beam Lorentz factor with increasing beam magnetization. This effect is less important in models having poloidal fields. Still, in all models except B1-pol-1 the mean magnetization in the beam drops below its initial value after the plasma has passed through successive recollimation shocks in the beam (in model B1-pol-1 the beam remains almost unperturbed). Even in the most strongly magnetized models the field never reaches equipartition values neither in the cross shocks nor in the hot spot. However, there might exist magnetic field configurations which lead to equipartition values at large distances from the nozzle, although this hypothesis has to be tested by simulations combining poloidal and toroidal fields.

If the magnetic field energy is indeed in equipartition with the internal energy of the plasma in the hotspot and other bright emission features of radio sources, then our results imply that the magnetic field triggering such features is either not mainly of toroidal nature or the initial magnetic field strength (at distances $\lesssim 1 \mathrm{kpc}$ from the AGN central engine) is well above equipartition (namely, $\bar{\beta} \gtrsim 100$ ). Otherwise, if observations point towards a toroidal structure of the magnetic field in bright radio features, the magnetic field energy might be more than one order of magnitude below equipartition in these features (if the magnetic field energy at distances $\lesssim 1 \mathrm{kpc}$ from the AGN core is in rough equipartition with the thermal energy). Both conclusions can explain the current estimates for the magnetic field strength in the hot spots of several radio galaxies which has values between a factor of 25 below equipartition and slightly above equipartition (e.g. Harris et al. 1994; Hardcastle et al. 1998, 2002; Wilson et al. 2000; Donahue et al. 2003).

The morphology and dynamics of relativistic jets can be substantially influenced by toroidal magnetic fields of a strength far below equipartition. This is demonstrated by model 
B1-1/20 which has a featureless cocoon as well as an optimal propagation efficiency and collimation. Since this behavior is not found in model C2-1/20 (and we have not simulated model C1-1/20), noticeable morphodynamical changes in jets carrying weak toroidal magnetic fields seem to require the concourse of several circumstances. Models B1-1/20 and C2-1/20 differ by the value of $\gamma$ (Table 1). As we have argued in Sect. 4.2.3 a smaller $\gamma$ yields a reduced lateral expansion of the cavity blown by the jet, which in turn increases the propagation speed of the head of the jet, and hence also increases the aspect ratio. If the jet propagates faster, the mass flux of the backflow is reduced. As the back flow is mainly responsible for exciting the Kelvin-Helmholtz modes in the surface of the beam, the reduced back flow in models having a relativistic (small) $\gamma$ reduces the amount of perturbations of the beam. A weak toroidal magnetic field in the beam helps to increase the collimation and propagation efficiency of the jet, because a small part of the mass that should flow backwards after crossing the Mach disk is forced into the nose cone and does not contribute to the upstream pinching of the beam. In model B1-1/20 the nose cone is reduced to a little plug in front of the Mach disk because the Lorentz force is small due to the small value of the field strength. The resulting tiny nose cone does not affect the propagation efficiency of the jet.

According to Begelman (1998) a cylindric jet carrying a purely toroidal magnetic field should be stable against pinching instabilities if

$\mathcal{E} \equiv \frac{\mathrm{d} \ln B^{\phi}}{\mathrm{d} \ln r}<\frac{\gamma-2 \beta}{\gamma+2 \beta}$

In the limit of slightly magnetized jets $(\beta \rightarrow 0)^{3}$ this expression approaches a value of one (i.e., the flow is stable if $\mathcal{E}<1$ ), while for highly magnetized beams $(\beta \rightarrow \infty)$ it tends to minus one (i.e., stability holds for $\mathcal{E}<-1$ ). The field in our jets is set up according to Eq. (44) which implies $\mathcal{E}=1$ for $r<r_{\mathrm{m}}$, and $\mathcal{E}=-1$ for $r \leq r_{\mathrm{m}}$, respectively. Hence, according to the linear stability analysis, the outer layer of the jet should be stable, while the inner part $\left(r<r_{\mathrm{m}}\right)$ should be unstable, independent of the degree of magnetization and the adiabatic index. We see the development of pinching modes in all jet models transporting toroidal magnetic fields. According to our interpretation model B1-1/20 is the most stable of all the models (although it develops some normal modes) because (for $\gamma=4 / 3$ and $\bar{\beta}=1 / 20$ ) $\mathcal{E}=0.86$, which is the value closest to the stability limit of all models. Indeed, for $\bar{\beta}_{\text {min }}<\bar{\beta}<1 / 20$, the jet might be even more stable than in case of model $\mathrm{B} 1-1 / 20$, but this trend should break down below some threshold $\bar{\beta}_{\text {min }}$, because model $\mathrm{B} 1-0$ is more unstable than $\mathrm{B} 1-1 / 20$.

The smaller value of the adiabatic index also explains why the nose cones of the models of series B1 $(\gamma=4 / 3)$ are smaller than those of the models of series C1 or C2 $(\gamma=5 / 3)$. The magnetization downstream of a strong shock depends on the adiabatic index through (Komissarov 1999b)

$\beta_{2}=6\left(\frac{\gamma_{1}}{\gamma_{1}-1} \frac{1}{2 \beta_{1}}+\frac{1}{\sigma_{1}}\right)^{-1}$,

${ }^{3}$ The expression is invalid for $\beta=0$. Therefore, we do not infer stability properties of the hydrodynamic models from Eq. (51). where the subscripts 1 and 2 refer to variables in the upstream and downstream state relative to the shock frame, respectively. Therefore, the magnetization downstream the Mach disk is smaller for smaller upstream $\gamma$. A reduced magnetization yields a smaller magnetic tension forcing the plasma into the plug, and hence less fluid will accumulate in the nose cone.

The evolution of axisymmetric jets can be divided into a $1 D$ phase and a 2D phase. During the initial 1D phase the evolution is almost linear in time, and the propagation velocity matches almost exactly the 1D estimate (Eq. (47)). This holds for the models of the series $\mathrm{C} 1$ and $\mathrm{C} 2$ despite their $p^{*}$ over-pressured beams (the 1D estimate is obtained for pressure matched hydrodynamic jets!), or their different working surface topologies. All models of series B1 propagate faster than estimated by Eq. (47) because of their adiabatic index is smaller $\gamma(=4 / 3)$. The $2 \mathrm{D}$ phase is characterized by the ejection of vortices from the head of the jet, and by the non-linear, multidimensional interaction between the beam and the cocoon. Usually during the 2D phase the propagation speed of the jet is reduced, because the head of the jet widens and the thrust is exerted onto a larger cross sectional area. The models of series B1 do not reach the 2D phase in our simulations. Their propagation velocity remains constant until the jets reach the end of the computational domain (Fig. 7). Models of series C1 and C2 reach, however, the $2 \mathrm{D}$ phase sufficiently early in their evolution. Especially in the models of series $\mathrm{C} 1$ the evolutionary times are very large, and the different phases are clearly distinguishable: the propagation velocities are constant and coincide with the estimated $1 \mathrm{D}$ velocity (Eq. (47)) up to $t \approx 50 r_{\mathrm{b}} / c$, where the jets start to decelerate. The aspect ratios become nearly constant at about the same time (Fig. 8, all models but C1-10/3). A similar behavior is observed for all models of series C2. Model C1-10/3 behaves differently, because its dynamics is completely dominated by the high density nose cone.

Models with poloidal fields develop remarkably smooth cocoons and jet cavities as well as very stable beams. Due to the configuration of the magnetic field no nose cones are formed. Instead, the beam plasma flows along the field lines which are bent sidewards by the expansion of the leading bow shock yielding overdense, hammer-like structures at the head of the jet (in models of series C). The cross shocks in the beam of jets threaded by poloidal fields are weaker than those having toroidal fields of the same average magnetization or even models without any magnetic field. This behavior is due to the magnetic tension in the beam that acts as a restoring force repelling waves driven into the beam. Owing to the smoothness of their beams relativistic jets with a dominant poloidal magnetic field component may have a brightness distribution along the beam which will be rather uniform in contrast to the very knotty distribution that we predict for jets with predominantly toroidal fields. However, the hot spot associated with the terminal shock of the jet might be similarly bright since the strength of the terminal shock is almost the same in the case of toroidal or poloidal field configurations. In jets with poloidal fields there is a substantial radial component of the magnetic field (directed towards the axis) at the bow shock (most evident in the models of series C; Figs. 5 and 16), which should manifest itself in radio maps of the polarized intensity. This feature might help 
to discriminate poloidal and toroidal magnetic field structures. The magnetic field is almost completely expelled from the cocoon of models with a poloidal field, but not from that of models with toroidal fields. Indeed, $\beta \approx 10^{-6}$ in most of the cavities of models having poloidal fields.

Our present approach has several limitations which prevent us from extracting more solid conclusions from our simulations. The evolutionary times covered in the simulations are very short (assuming $r_{\mathrm{b}} \sim 0.3 \mathrm{kpc}$ the longest evolutionary time is $t_{\max } \sim 2.5 \times 10^{5} \mathrm{y}$ corresponding to the series $\mathrm{C} 1$ ) compared to the typical lifetimes of extragalactic radio sources $\left(\approx 10^{7}-10^{8} \mathrm{y}\right)$. The size of the domain in the direction perpendicular to the jet axis is not sufficiently large as to contain the complete cocoon in some of the simulations. Both technical limitations will be overcome in a forthcoming paper where we will consider the long-term evolution of kiloparsec scale jets transporting toroidal fields in a sufficiently large computational box. Furthermore, three-dimensional (3D) simulations are necessary in order to include all the possible destabilizing properties of the magnetic field and to study the stability properties of relativistic magnetized jets. This will be addressed in a future investigation. As 3D simulations are obviously very costly, our present comprehensive parameter study of axisymmetric RMHD jets serves to identify and to select the most significant cases to be simulated later in 3D without any symmetry restriction.

Finally, we point out that we have presented a new highorder numerical algorithm to integrate the RMHD equations in several spatial dimensions for flows of astrophysical interest. The verification and robustness of the algorithm has been proven with several one- and two-dimensional tests the results being qualitatively the same as those produced with other existing numerical codes.

Acknowledgements. The numerical simulations presented here were carried out on the IBM p690 Regatta computer of the Rechenzentrum Garching (RZG), and we gratefully acknowledge the RZG for providing that computer time. M.A.A. acknowledges support through an agreement between the Max-Planck-Gesellschaft and the Consejo Superior de Investigaciones Científicas. L.A. acknowledges the support received by the Spanish DGES through grant PB97-1432. This research was supported in part by the Spanish Dirección General de Enseñanza Superior grants AYA-2001-3490-C02-01 and AYA-20013490-C02-02.

\section{References}

Abramowitz, M., \& Stegun, A. 1965, Handbook of Mathematical Functions (New York: Dover)

Aloy, M., Martí, J., Gómez, J., et al. 2003, ApJ, 585, L109

Aloy, M. A., Gómez, J.-L., Ibáñez, J. M., Martí, J. M., \& Müller, E. 2000, ApJ, 528, L85

Aloy, M. A., Ibáñez, J. M., Martí, J. M., Gómez, J.-L., \& Müller, E. 1999a, ApJ, 523, L125

Aloy, M. A., Ibáñez, J. M., Martí, J. M., \& Müller, E. 1999b, ApJS, 122,151

Anile, A. M. 1989, Relativistic fluids and magneto-fluids (Cambridge University Press)

Appl, S., \& Camenzind, M. 1988, A\&A, 206, 258
Attridge, J. M., Roberts, D. H., \& Wardle, J. F. C. 1999, ApJ, 518, L87

Balsara, D. S. 2001, ApJS, 132, 83

Begelman, M. C. 1998, ApJ, 493, 291

Begelman, M. C., \& Cioffi, D. F. 1989, ApJ, 345, L21

Begelmann, M. C., Blandford, R. D., \& Rees, M. J. 1984, Rev. Mod. Phys., 56, 255

Brio, M., \& Wu, C.-C. 1988, J. Comp. Phys., 75, 400

Bucciantini, N., Amato, E., \& Del Zanna, L. 2004

[arXiv: astro-ph/0412534], A\&A, to appear

Clarke, D. A., Norman, M. L., \& Burns, J. O. 1986, ApJ, 311, L63

Crusius-Waetzel, A. R., Biermann, P. L., Schlickeiser, R., \& Lerche, I. 1990, ApJ, 360, 417

De Villiers, J.-P., \& Hawley, J. F. 2003, ApJ, 592, 1060

De Villiers, J.-P., \& Hawley, J. F. 2003, ApJ, 589, 458

Del Zanna, L., Amato, E., \& Bucciantini, N. 2004, A\&A, 421, 1063

Del Zanna, L., Bucciantini, N., \& Londrillo, P. 2003, A\&A, 400, 397

Donahue, M., Daly, R. A., \& Horner, D. J. 2003, ApJ, 584, 643

Donat, R., \& Marquina, A. 1996, J. Comp. Phys., 125, 42

Dubal, M. 1991, Comp. Phys. Comm., 64, 221

Evans, C. R., \& Hawley, J. F. 1988, ApJ, 332, 659

Ferrari, A. 1998, ARA\&A, 36, 539

Gabuzda, D. C. 2003, New Astron. Rev., 47, 599

Gabuzda, D. C., \& Cawthorne, T. V. 1996, MNRAS, 283, 759

Gabuzda, D. C., \& Gómez, J. L. 2001, MNRAS, 320, L49

Gabuzda, D. C., \& Pushkarev, A. B. 2001, in Particles and Fields in Radio Galaxies Conference, ed. R. A. Laing \& K. M. Blundell, ASP Conf. Proc., ASP Conf. Ser., 250, 180

Gabuzda, D. C., Pushkarev, A. B., \& Cawthorne, T. V. 2000, MNRAS, 319,1109

Gabuzda, D. C., Pushkarev, A. B., \& Garnich, N. N. 2001, MNRAS, 327,1

Gammie, C. F., McKinney, J. C., \& Tóth, G. 2003, ApJ, 589, 444

Hardcastle, M. J., Birkinshaw, M., Cameron, R. A., et al. 2002, ApJ, 581,948

Hardcastle, M. J., Birkinshaw, M., \& Worrall, D. M. 1998, MNRAS, 294, 615

Harris, D. E., Carilli, C. L., \& Perley, R. A. 1994, Nature, 367, 713

Harten, A., Lax, P. D., \& van Leer, B. 1983, SIAM Rev., 25, 35

Kigure, H., Uchida, Y., Nakamura, M., Hirose, S., \& Cameron, R. 2004, ApJ, 608, 119

Kim, K.-T., Kronberg, P. P., Dewdney, P. E., \& Landecker, T. L. 1990, ApJ, 355, 29

Koide, S. 1997, ApJ, 478, 66

Koide, S. 2003, Phys. Rev. D, 67, 104010

Koide, S., Meier, D. L., Shibata, K., \& Kudoh, T. 2000, ApJ, 536, 668

Koide, S., Nishikawa, K., \& Mutel, R. L. 1996, ApJ, 463, L71

Koide, S., Shibata, K., \& Kudoh, T. 1998, ApJ, 495, L63

Koide, S., Shibata, K., \& Kudoh, T. 1999, ApJ, 522, 727

Koide, S., Shibata, K., Kudoh, T., \& Meier, D. L. 2002, Science, 295 , 1688

Koldoba, A. V., Kuznetsov, O. A., \& Ustyugova, G. V. 2002, MNRAS, 333, 932

Komissarov, S. S. 1999a, MNRAS, 303, 343

Komissarov, S. S. 1999b, MNRAS, 308, 1069

Komissarov, S. S., \& Lyubarsky, Y. E. 2003, MNRAS, 344, L93

Komissarov, S. S., \& Lyubarsky, Y. E. 2004, MNRAS, 349, 779

Königl, A. 1980, Phys. Fluids, 23, 1083

Kössl, D., Müller, E., \& Hillebrandt, W. 1990, A\&A, 229, 378

Krause, M. 2003, A\&A, 398, 113

Krause, M., \& Löhr, A. 2004, A\&A, 420, 115

Laing, R. A., \& Bridle, A. H. 2002, MNRAS, 336, 328

Landau, L. D., \& Lifshitz, E. M. 1966, Lehrbuch der Theoretischen Physik, Vol. VI, Hydrodynamik (Berlin: Akademie Verlag) 
Leismann, T., Aloy, M., \& Müller, E. 2004, Ap\&SS, 293, 157

LeVeque, R. J. 1991, Numerical Methods for Conservation Laws (Basel: Birhkhäuser)

Lind, K. R., Payne, D. G., Meier, D. L., \& Blandford, R. D. 1989, ApJ, 344,89

Londrillo, P., \& Del Zanna, L. 2004, J. Comp. Phys., 195, 17

Lovelace, R. V. E., Li, H., Koldoba, A. V., Ustyugova, G. V., \& Romanova, M. M. 2002, ApJ, 572, 445

Lynden-Bell, D. 2003, MNRAS, 341, 1360

Martí, J. M., \& Müller, E. 1996, J. Comp. Phys., 123, 1

Martí, J. M., Müller, E., Font, J. A., Ibáñez, J. M., \& Marquina, A. 1997, ApJ, 479, 151

Mizuno, Y., Yamada, S., Koide, S., \& Shibata, K. 2004a, ApJ, 606, 395

Mizuno, Y., Yamada, S., Koide, S., \& Shibata, K. 2004b, ApJ, 615, 389

Nakamura, M., Uchida, Y., \& Hirose, S. 2001, New Astron., 6, 61

Nishikawa, K., Koide, S., Sakai, J., et al. 1997, ApJ, 483, L45

Nishikawa, K., Koide, S., Sakai, J., et al. 1998, ApJ, 498, 166

Nishikawa, K., Koide, S., Shibata, K., et al. 2002, APS Meeting Abstracts, 17093

Nishikawa, K.-I., Richardson, G., Preece, R., et al. 2003, BAAS, 202, 602
Parker, E. N. 1979, Cosmical magnetic fields: Their origin and their activity (New York, Oxford: Oxford, Clarendon Press; University Press), 858

Press, W. H., Teukolsky, S., \& Vetterling, W. T. 1992, Numerical Recipes in FORTRAN, 2nd ed. (Cambridge University Press)

Pushkarev, A. B., \& Gabuzda, D. C. 2001, in Particles and Fields in Radio Galaxies (San Francisco: ASP), ed. R. A. Laing \& K. M. Blundell, ASP Conf. Proc., 250, 200

Ryu, D., Miniati, F., Jones, T. W., \& Frank, A. 1998, ApJ, 509, 244

Scheck, L., Aloy, M. A., Martí, J. M., Gómez, J. L., \& Müller, E. 2002, MNRAS, 331, 615

Shu, C. W., \& Osher, S. J. 1988, J. Comp. Phys., 77, 439

Swain, M. R., Bridle, A., \& A., B. S. 1998, ApJ, 507, L29

Taylor, G. B., \& Perley, R. A. 1993, ApJ, 416, 554

Tsinganos, K., \& Bogovalov, S. 2002, MNRAS, 337, 553

van Putten, M. H. P. M. 1993, ApJ, 408, L21

van Putten, M. H. P. M. 1995, SIAM J. Num. Anal., 32, 1504

van Putten, M. H. P. M. 1996, ApJ, 467, L57

Walker, R. C., Benson, J. M., \& Unwin, S. C. 1987, ApJ, 316, 546

Wilson, A. S., Young, A. J., \& Shopbell, P. L. 2000, ApJ, 544, L27 


\section{Online Material}




\section{Appendix A: The equations of RMHD in cylindrical coordinates}

For jet applications where axisymmetry is assumed, Eqs. (1), (2) and (10) have to be written in cylindrical coordinates, i.e., the corresponding vector of geometrical source terms has be added to the right hand side of the equations, $\boldsymbol{Q}$, and the appropriate geometrical factors have to be considered.

Using cylindrical coordinates $(r, \phi, z)$ the metric becomes

$g_{\alpha \beta}=\operatorname{diag}\left(-1,1, r^{2}, 1\right)$.

Then the mass conservation Eq. (1) reads

$\partial_{0} D+\frac{1}{r} \partial_{r}\left(r D v^{r}\right)+\frac{1}{r} \partial_{\phi}\left(D v^{\phi}\right)+\partial_{z}\left(D v^{z}\right)=0$,

and the momentum and energy conservation Eqs. (2) become

$$
\begin{aligned}
\partial_{0} S^{r} & +\frac{1}{r} \partial_{r}\left[r\left(\rho h^{*} W^{2} v^{r} v^{r}+p^{*}-b^{r} b^{r}\right)\right] \\
& +\frac{1}{r} \partial_{\phi}\left(\rho h^{*} W^{2} v^{r} v^{\phi}-b^{r} b^{\phi}\right) \\
& +\partial_{z}\left(\rho h^{*} W^{2} v^{r} v^{z}-b^{r} b^{z}\right)=\frac{\rho h^{*} W^{2} v^{\phi} v^{\phi}+p^{*}-b^{\phi} b^{\phi}}{r} \\
\partial_{0} S^{z} & +\frac{1}{r} \partial_{r}\left[r\left(\rho h^{*} W^{2} v^{z} v^{r}-b^{r} b^{z}\right)\right] \\
& +\frac{1}{r} \partial_{\phi}\left(\rho h^{*} W^{2} v^{z} v^{\phi}-b^{z} b^{\phi}\right) \\
& +\partial_{z}\left(\rho h^{*} W^{2} v^{z} v^{z}+p^{*}-b^{z} b^{z}\right)=0 \\
\partial_{0} S^{\phi} & +\frac{1}{r} \partial_{r}\left[r\left(\rho h^{*} W^{2} v^{\phi} v^{r}-b^{\phi} b^{r}\right)\right] \\
& +\frac{1}{r} \partial_{\phi}\left(\rho h^{*} W^{2} v^{\phi} v^{\phi}+p^{*}-b^{\phi} b^{\phi}\right) \\
& +\partial_{z}\left(\rho h^{*} W^{2} v^{\phi} v^{z}-b^{\phi} b^{z}\right)=-\frac{\rho h^{*} W^{2} v^{r} v^{\phi}-b^{r} b^{\phi}}{r} \\
& +\frac{1}{r} \partial_{r}\left[r\left(\rho h^{*} W^{2} v^{r}-b^{r} b^{0}-D v^{r}\right)\right] \\
& +\frac{1}{r} \partial_{\phi}\left(\rho h^{*} W^{2} v^{\phi}-b^{\phi} b^{0}-D v^{*}\right) \\
\partial_{0} \tau &
\end{aligned}
$$

Accordingly, written component-wise, the induction Eq. (10) in cylindrical coordinates reads

$$
\begin{gathered}
\partial_{0} B^{r}+\partial_{z}\left(v^{z} B^{r}-v^{r} B^{z}\right)+\frac{1}{r} \partial_{\phi}\left(v^{\phi} B^{r}-v^{r} B^{\phi}\right)=0 \\
\partial_{0} B^{z}+\frac{1}{r} \partial_{r}\left[r\left(v^{r} B^{z}-v^{z} B^{r}\right)\right]+\frac{1}{r} \partial_{\phi}\left(v^{\phi} B^{z}-v^{z} B^{\phi}\right)=0 \\
\partial_{0} B^{\phi}+\frac{1}{r} \partial_{r}\left[r\left(v^{r} B^{\phi}-v^{\phi} B^{r}\right)\right]+\partial_{z}\left(v^{z} B^{\phi}-v^{\phi} B^{z}\right)= \\
\frac{B^{\phi} v^{r}-B^{r} v^{\phi}}{r}
\end{gathered}
$$

Equations (A.2)-(A.9) cast the RMHD system into cylindrical coordinates, where geometrical source terms occur in the equations for the $r$ - and $\phi$-momentum, and for the $\phi$ magnetic field (note that the vector components are given in the Cylindric orthonormal basis). Accordingly the vector of source terms is given by

$$
\begin{aligned}
\boldsymbol{Q}= & \left(0, \frac{\rho h^{*} W^{2} v^{\phi} v^{\phi}+p^{*}-b^{\phi} b^{\phi}}{r}, 0,\right. \\
& \left.-\frac{\rho h^{*} W^{2} v^{r} v^{\phi}-b^{r} b^{\phi}}{r}, 0,0,0, \frac{B^{\phi} v^{r}-B^{r} v^{\phi}}{r}\right)^{T} .
\end{aligned}
$$

\section{Appendix B: Code verification}

Before a numerical simulation code is used to solve a physical problem, it is necessary to verify its results by comparing them with known solutions of test problems. To this end one sets up several Riemann problems in one or more dimensions and checks whether the code can resolve all the waves that result from the breakup of the initial discontinuity. Ideal test cases would be those where an analytical solution to the problem is known. However, in RMHD there are not too many tests with known solutions mainly because a closed solution for the general RMHD Riemann problem has not yet been found. An alternative way of testing a code is to cross-check its test results against those obtained by other authors for the same test problems. With this aim in mind we have verified our code against the 1D test problems from Balsara (2001) which are also reproduced by Del Zanna et al. (2003). In 2D we have used the results of Komissarov (1999a).

\section{B.1. $1 D$ test problems}

Table B.1 lists the parameters of the five 1D Riemann problems described in Balsara (2001). The first four were also considered by Del Zanna et al. (2003). Every test involves a discontinuity placed in the center of a 1D computational domain of 1600 grid zones. We ran each of the five problems twice: (1) using PLM interpolation with a threshold of $\beta=4$ for the slope limiting (see Sect. 2.3.2); and (2) using PPM. A Courant number of 0.5 was used in all of the test runs. For the sake of conciseness, and considering that our results are of the same quality as those of Balsara (2001) and Del Zanna et al. (2003) we only show here tests 3 and 4 of Table B.1.

The results of the blast wave test problems 3 is displayed in Fig. B.1. Again the resulting waves are the same as in Balsara (2001). Test 3 is a strong blast wave with a large initial pressure difference. It develops two rarefaction waves propagating to the left, for example displayed in the density and pressure panels of Fig. B.1, which are both captured equally well by PPM and PLM. However, the high density structure on the right is neither resolved in our runs nor in Balsara (2001). Nevertheless, using PLM and PPM we obtain the same maximum Lorentz factor of about 3.4 as Balsara (2001). This illustrates that despite the numerical viscosity of the algorithm we can obtain the physically correct overall structure for this test problem. The comparison with Del Zanna et al. (2003) shows that our PPM results are again slightly better resolved in test No. 2 (not shown in this paper), while we get slightly worse results for the stronger blast wave test No. 3. This is visible from the height 
Table B.1. Parameters for the 1D Riemann problems.

\begin{tabular}{llrrrrrrrrrr}
\hline \hline No. & Initial state & $\rho$ & $p$ & $v_{x}$ & $v_{y}$ & $v_{z}$ & $B_{y}$ & $B_{z}$ & $B_{x}$ & $\gamma$ & $t_{\text {final }}$ \\
\hline 1 & left & 1.0 & 1.0 & 0.0 & 0.0 & 0.0 & 1.0 & 0.0 & 0.5 & 2.0 & 0.4 \\
& right & 0.125 & 0.1 & 0.0 & 0.0 & 0.0 & -1.0 & 0.0 & 0.5 & 2.0 & 0.4 \\
\hline 2 & left & 1.0 & 30.0 & 0.0 & 0.0 & 0.0 & 6.0 & 6.0 & 5.0 & $5 / 3$ & 0.4 \\
& right & 1.0 & 1.0 & 0.0 & 0.0 & 0.0 & 0.7 & 0.7 & 5.0 & $5 / 3$ & 0.4 \\
\hline 3 & left & 1.0 & 1000.0 & 0.0 & 0.0 & 0.0 & 7.0 & 7.0 & 10.0 & $5 / 3$ & 0.4 \\
& right & 1.0 & 0.1 & 0.0 & 0.0 & 0.0 & 0.7 & 0.7 & 10.0 & $5 / 3$ & 0.4 \\
\hline 4 & left & 1.0 & 0.1 & 0.999 & 0.0 & 0.0 & 7.0 & 7.0 & 10.0 & $5 / 3$ & 0.4 \\
& right & 1.0 & 0.1 & -0.999 & 0.0 & 0.0 & -7.0 & -7.0 & 10.0 & $5 / 3$ & 0.4 \\
\hline 5 & left & 1.08 & 0.95 & 0.4 & 0.3 & 0.2 & 0.3 & 0.3 & 2.0 & $5 / 3$ & 0.55 \\
& right & 1.0 & 1.0 & -0.45 & -0.2 & 0.2 & -0.7 & 0.5 & 2.0 & $5 / 3$ & 0.55 \\
\hline
\end{tabular}
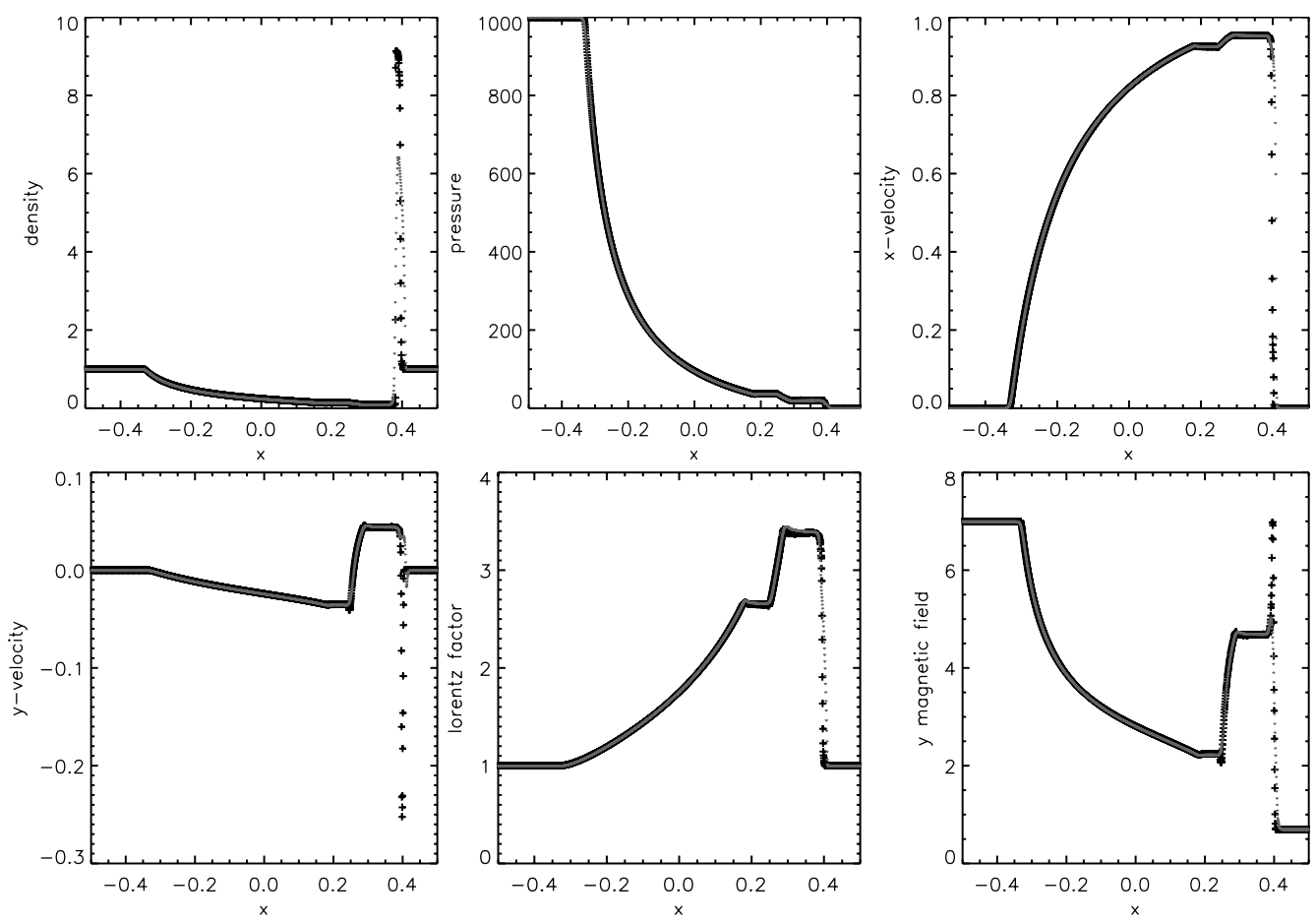

Fig. B.1. Third 1D test problem. Blast wave test with large initial pressure difference. The black crosses are the results obtained with PPM interpolation, the grey dots those computed with PLM interpolation.

of the high density shell which is $40 \%$ larger for PPM than for PLM, but approximately $2 \%$ smaller than in Del Zanna et al. (2003).

Figure B. 2 shows the results of test No.4, a strong relativistic shock reflection test with two streams approaching each other at a Lorentz factors of 22.366. This setup produces two fast and two slow shocks. Both PPM and PLM handle this test very well, but PLM requires more zones to resolve the slow shocks. At the initial collision point $(x=0)$ a certain amount of "wall heating" occurs, which is a numerical pathology of approximate Riemann solvers (e.g. Donat \& Marquina 1996). The problem arises because an excess of entropy is generated at the collision point at $t=0$. This can diffuse numerically only slowly, because the fluid is at rest at that point. Higher order reconstruction schemes help to confine the problem to a small number of points initially, but generate less diffusion. Hence the "hole" in the rest mass density is larger with PPM (confined to only three grid zones with a relative error of about 10\%) than with PLM (the "hole" is much more spread out with an error of 5\%). In this test our PLM results are of similar quality than those of Balsara (2001) and Del Zanna et al. (2003), while our PPM implementation requires less zones to resolve the slow shocks.

In summary, our code solves all test problems presented in Balsara (2001) correctly. As a rule of thumb, the PPM runs require less zones to resolve structures (in particular those of slowly moving waves) than the methods described in Balsara (2001) and Del Zanna et al. (2003), which are closer to our PLM results.

\section{B.2. $2 D$ test problems}

While the 1D tests have shown that our code can resolve all the waves appearing in RMHD reasonably well, 2D calculations introduce further complexities related with the constraint that the divergence of the magnetic field should 
T. Leismann et al.: RMHD simulations of extragalactic jets, Online Material p 4
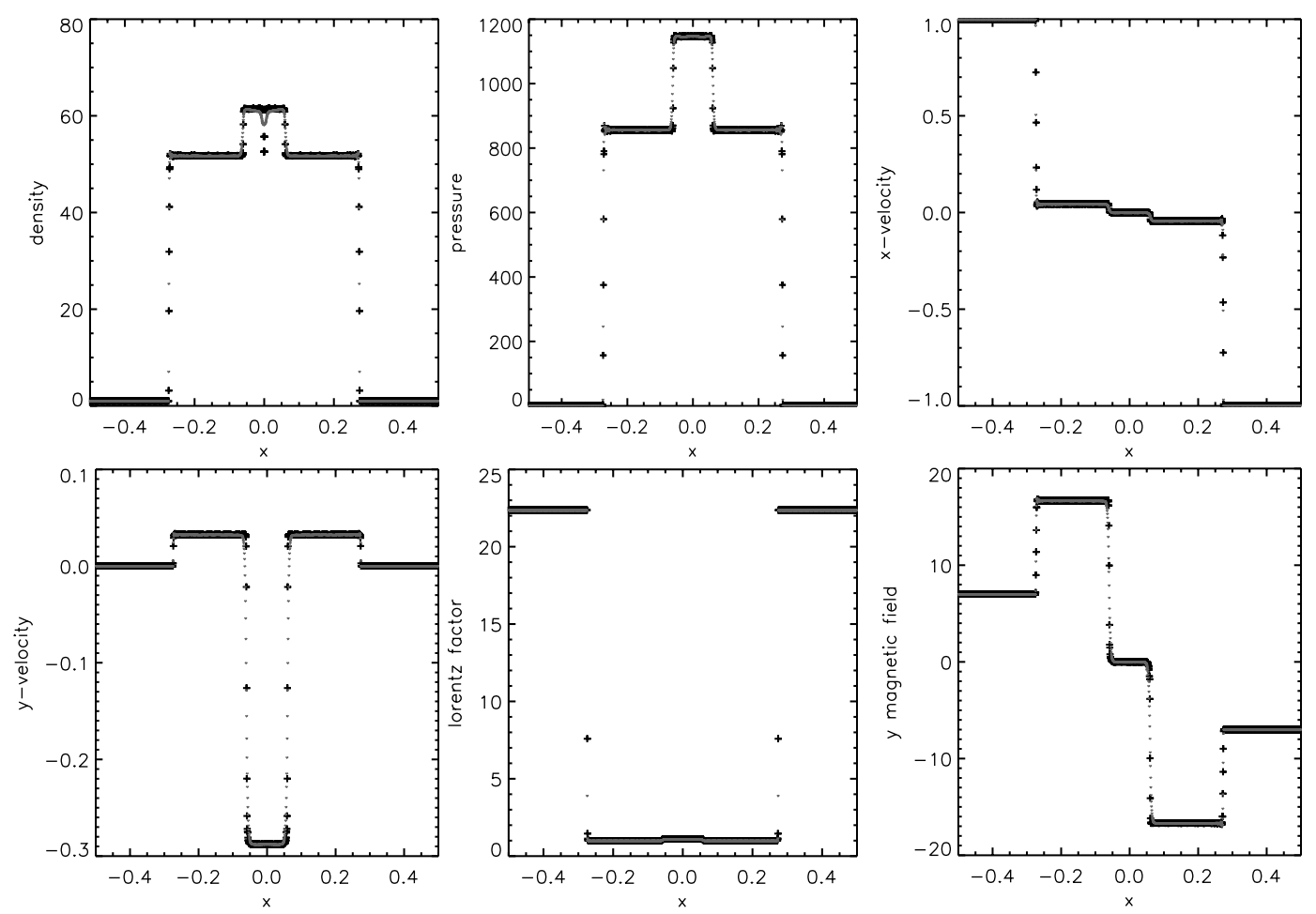

Fig. B.2. Fourth 1D test problem. Shock reflection test. The black crosses are the results obtained with PPM interpolation, the grey dots those computed with PLM interpolation.

remain zero (Sect. 2.3.4), which is trivially fulfilled in $1 \mathrm{D}$ tests. Furthermore, in Sect. B.2.2 we include an standard convergence test in $2 \mathrm{D}$ which shows that our algorithm converges at global second order accuracy.

\section{B.2.1. Cylindrical explosion test}

The cylindrical explosion test consists of a strong shock propagating into a magnetically dominated medium. Although there is no analytic solution to verify against, this test still has very useful properties which make it a standard test of multidimensional numerical schemes for gas dynamics and MHD. For example, it encompasses all the degeneracies of the RMHD eigensystem (Sect. 2.2.1). Its simple setting makes it easy to spot bugs or weaknesses of a scheme which might not be seen so clearly in more complicated problems. Results for different cylindrical explosion problems in RMHD have been published (1) by Dubal (1991) indicating severe problems with his scheme; (2) by van Putten (1995) reaching a maximum velocity of $v=0.35$; (3) by Komissarov (1999a); and (4) by Del Zanna et al. (2003).

We have chosen a setup very similar to that in Komissarov (1999a): a cylinder of high pressure and density is located in the center of a square Cartesian grid, which initially contains a uniform, strong magnetic field. The grid has 200 by 200 zones spanning 12 by 12 units of distance. In the center of the grid there is a circle of radius 0.8 where $\rho=10^{-2}$ and $p=1$. Between a radius of 0.8 and 1.0 the values smoothly decrease to those of the homogeneous ambient medium $\left(\rho=10^{-4}\right.$ and $\left.p=5 \times 10^{-4}\right)$. Initially, the magnetic field is $B^{x}=0.1$, and the velocity is zero everywhere. The simulations were carried out on an IBM Power4 processor and required about $300 \mathrm{~s}$ of CPU time.

Figure B. 3 shows the results of this test at time $t=4.0$ using PLM. One recognizes an outer fast shock, which is almost circular, because the fast magnetosonic speed varies little across the grid. The innermost region is also circular and bounded by a reverse fast shock. The expansion of this region is almost circular, because the Lorentz force is small there. In between these two shocks there are two more discontinuities bounded on the outside by the compressed magnetic field (see the field lines in the Lorentz factor panel of Fig. B.3). The CT method works in both cases as demonstrated by the plot of $\nabla \cdot \boldsymbol{B}$ (the non-zero values are consistent with machine accuracy in double precision FORTRAN arithmetics).

The PPM results exhibit oscillations on a $5 \%$ level which are largely reduced by increasing the resolution. Figure B.4 shows plots of the thermal pressure along $x=0$ for two different resolutions: 200 by 200 zones (upper panel) and 800 by 800 zones (lower panel). While PPM (black crosses) requires less points than PLM (grey dots) in discontinuities in the higher resolution run, there is no such trend in the lower resolution run. At both resolutions PPM produces small oscillations.

\section{B.2.2. Convergence test}

In order to show that our algorithm, specially in the multidimensional case, is able to preserve global second order accuracy, we include a convergence test that has been also used in previous RMHD algorithms for the same purpose (Del Zanna et al. 2003). The test consists on the propagation of relativistic circularly polarized Alfvén waves. Our set up coincides with 
T. Leismann et al.: RMHD simulations of extragalactic jets, Online Material p 5
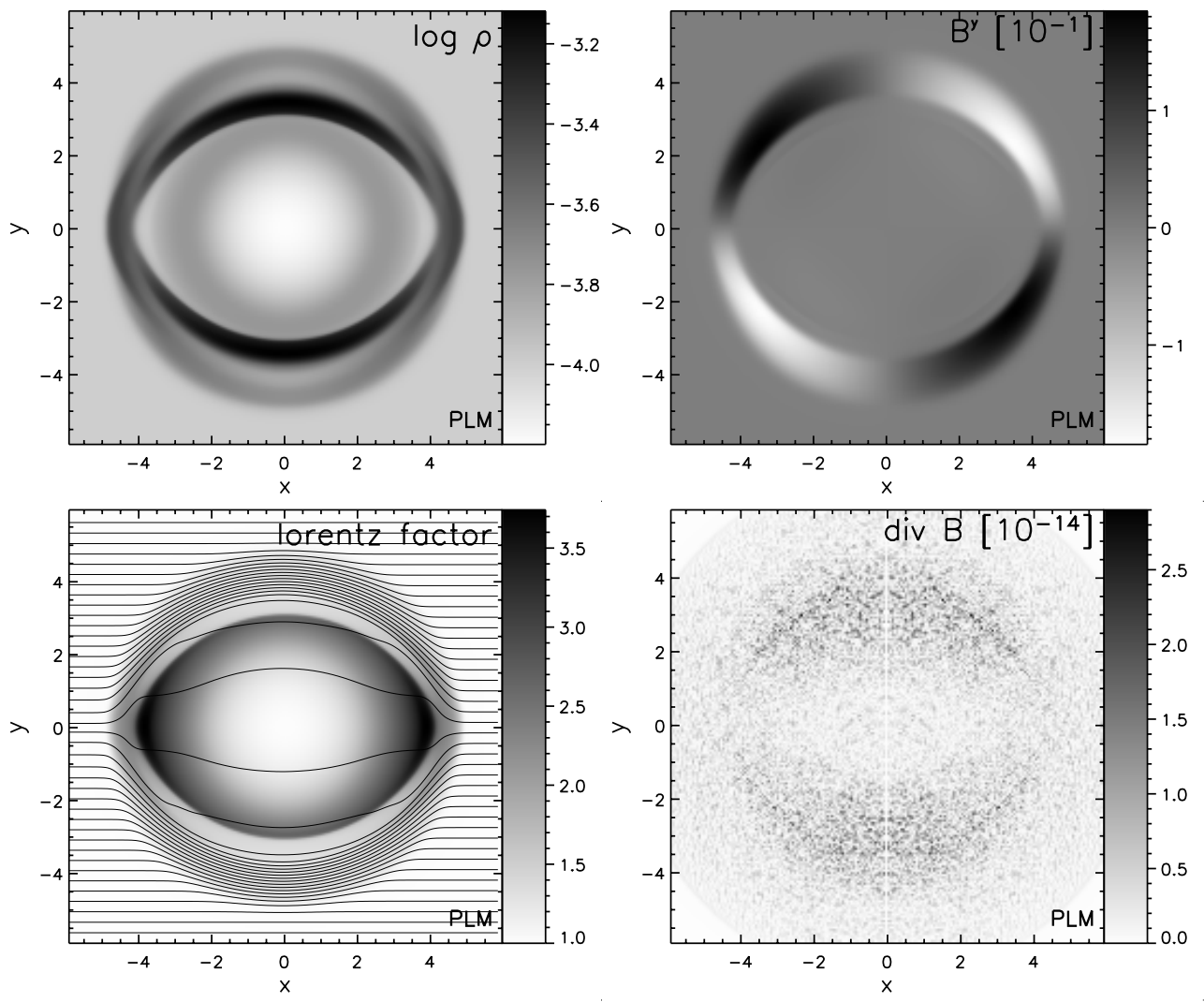

Fig. B.3. Cylindrical explosion test with PLM reconstruction.

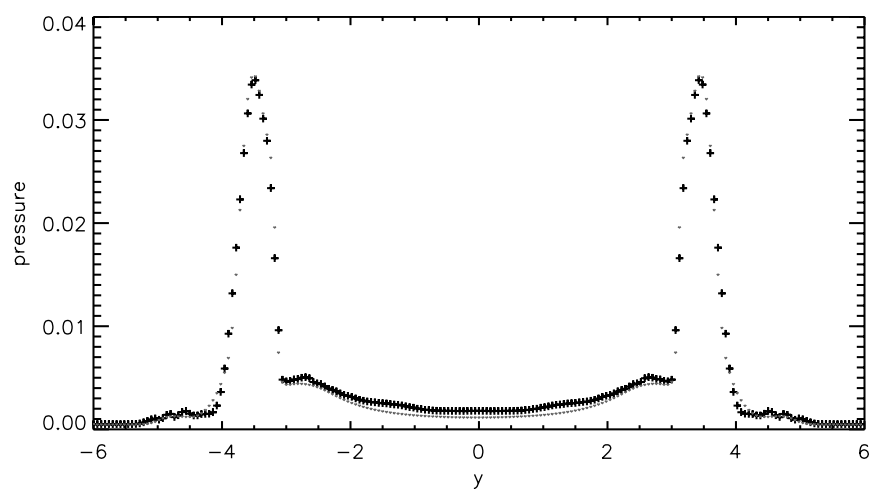

that of Del Zanna et al. (2003), i.e., after one period $T$ we evaluate the $L_{1}$ norm of the numerical solution, compared to the initial values,

$L_{1}(A)=\frac{\sum_{i j}\left|A\left(x_{i}, y_{j} ; t=T\right)-A\left(x_{i}, y_{j} ; t=0\right)\right|}{\sum_{i j}\left|A\left(x_{i}, y_{j} ; t=0\right)\right|}$,

where $A$ can be any variable. In order to compare directly with other authors, we choose $A=v_{z}$. The results are displayed in Fig. B.5, where the PLM reconstruction is used. It is easy to see that the algorithm converges at global second order. A similar test using the PPM reconstruction shows that the relative

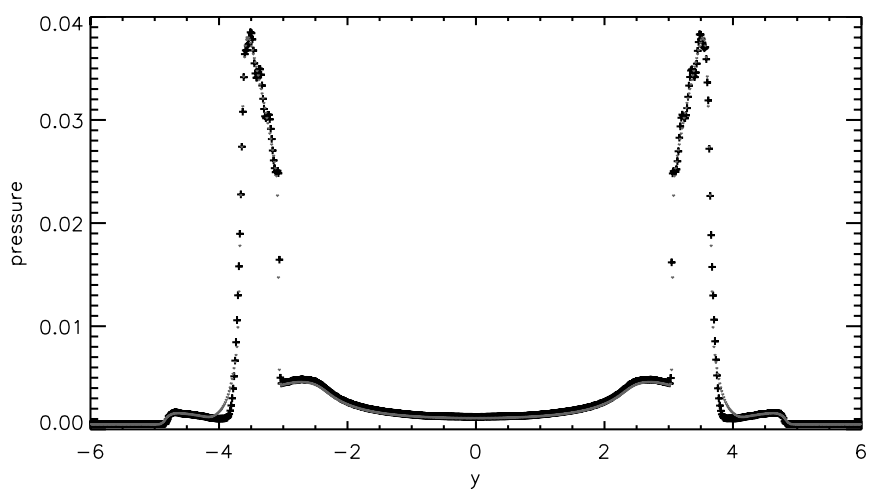

Fig. B.4. Plot along $x=0$ of the thermal pressure of the cylindrical explosion test. The top panel shows the results for the original grid of 200 by 200 zones, while the bottom panel is the result of the same test on a grid of 800 by 800 zones. The black crosses are obtained with PPM interpolation, the grey dots with PLM interpolation. errors are smaller at any given resolution than using PLM but the global order of convergence is the same.

\section{Appendix C: Proof of $\left|\lambda_{\mathrm{fms}}^{ \pm}\right| \leq\left|\lambda_{\mathrm{deg}, \mathrm{fms}}^{ \pm}\right|$}

The quartic equation that yields the magnetosonic eigenvalues can be cast in the following form

$\rho h\left(\frac{1}{c_{s}^{2}}-1\right) a^{4}-\left(\rho h+\frac{\boldsymbol{b}^{2}}{c_{s}^{2}}\right) a^{2} G+\mathcal{B}^{2} G=0$,

where $G \equiv 1-\lambda^{2}, \mathcal{B} \equiv b^{x}-\lambda b^{0}$ and $a \equiv u^{x}-\lambda u^{0}$. Equivalently, we can write

$\left(\frac{\mathcal{B}}{a}\right)^{2}=\left(\rho h+\frac{\boldsymbol{b}^{2}}{c_{s}^{2}}\right)-\rho h\left(\frac{1}{c_{s}^{2}}-1\right) \frac{a^{2}}{G}$. 


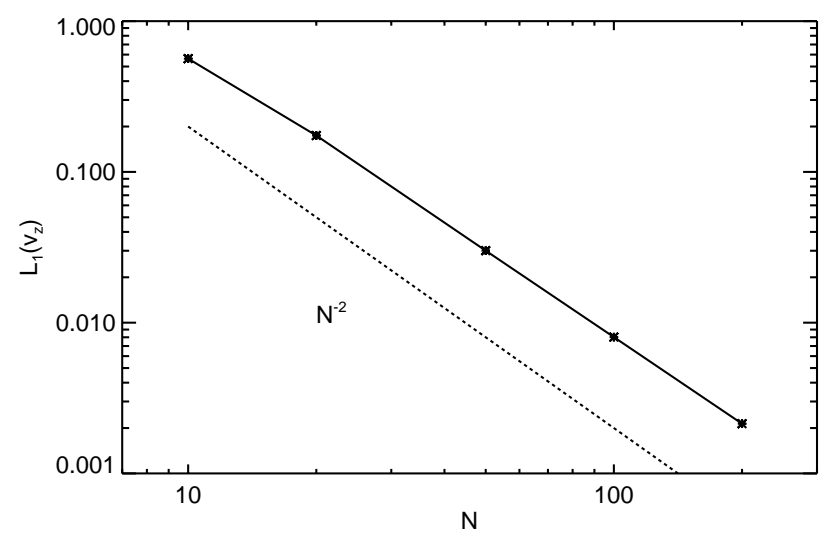

Fig. B.5. Convergence test for the propagation of circularly polarized Alfvén waves in 2D. The relative $L_{1}$ errors (Eq. B.1) are shown in logarithmic scale (solid line) as a function of the resolution per dimension $\left(N=N_{x}=N_{y}\right)$. The PLM reconstruction has been used. As a reference, the perfect second order convergence slope is also shown (dashed line).

Since all eigenvalues have to be real numbers, both $\mathcal{B}$ and $a$ must be real, and hence the right hand side of Eq. (C.2) has to be non negative, i.e.,

$\rho h+\frac{\boldsymbol{b}^{2}}{c_{s}^{2}}-\rho h\left(\frac{1}{c_{s}^{2}}-1\right) \frac{a^{2}}{G} \geq 0$.

After some algebra, Eq. (C.3) leads to the following quadratic inequality

$$
\begin{aligned}
-\lambda^{2}\left(\omega^{2}+\left(1-\omega^{2}\right)\left(u^{0}\right)^{2}\right) & +2 \lambda u^{0} u^{x}\left(1-\omega^{2}\right) \\
& +\omega^{2}-\left(1-\omega^{2}\right)\left(u^{x}\right)^{2} \geq 0
\end{aligned}
$$

This inequality holds for every real magnetosonic eigenvalue in the interval bounded by the roots

$$
\lambda_{\mathrm{r}}^{ \pm}=\frac{u^{0} u^{x}\left(1-\omega^{2}\right) \pm \sqrt{\omega^{2}\left(\omega^{2}+\left(1-\omega^{2}\right)\left(\left(u^{0}\right)^{2}-\left(u^{x}\right)^{2}\right)\right)}}{\omega^{2}+\left(1-\omega^{2}\right)\left(u^{0}\right)^{2}},
$$

i.e., the inequality implies $\left|\lambda_{\mathrm{fms}}^{ \pm}\right| \leq\left|\lambda_{\mathrm{r}}^{ \pm}\right|$.

It turns out that the fast magnetosonic eigenvalues for the degenerate case $B^{x}=0$ and $b^{0}=0$ are exactly equal to $\lambda_{\mathrm{r}}^{ \pm}$(see Eq. (27)), and therefore $\lambda_{\mathrm{fms}}^{ \pm} \leq \lambda_{\mathrm{deg}, \mathrm{fms}}^{ \pm}$. 